\title{
Neoadjuvant immunotherapy or chemoimmunotherapy in non-small cell lung cancer: a systematic review and meta-analysis
}

\author{
Juan Jiang ${ }^{1,2,3,4,5,6}$, Yuling Wang ${ }^{1}$, Yang Gao ${ }^{2,6,7}$, Haruhiko Sugimura ${ }^{8}$, Fabrizio Minervini ${ }^{9}$, Junji Uchino ${ }^{10,11}$, \\ Balazs Halmos $^{12}$, Sai Yendamuri ${ }^{13}$, Jeffrey B. Velotta ${ }^{14}$, Min Li $^{1,2,3,4,5,6}$ \\ ${ }^{1}$ Department of Respiratory Medicine, National Key Clinical Specialty, Branch of National Clinical Research Center for Respiratory Disease, \\ Xiangya Hospital, Central South University, Changsha, China; ${ }^{2}$ Xiangya Lung Cancer Center, Xiangya Hospital, Central South University, \\ Changsha, China; ${ }^{3}$ Center of Respiratory Medicine, Xiangya Hospital, Central South University, Changsha, China; ${ }^{4}$ Clinical Research Center \\ for Respiratory Diseases in Hunan Province, Changsha, China; ${ }^{5}$ Hunan Engineering Research Center for Intelligent Diagnosis and Treatment \\ of Respiratory Disease, Changsha, China; 'National Clinical Research Center for Geriatric Disorders, Xiangya Hospital, Changsha, China; \\ ${ }^{7}$ Department of Thoracic Surgery, Xiangya Hospital, Central South University, Changsha, China; ${ }^{8}$ Department of Tumor Pathology, Hamamatsu \\ University School of Medicine, Hamamatsu, Shizuoka, Japan; ${ }^{9}$ Department of Thoracic Surgery, Cantonal Hospital Lucerne, Lucerne, Switzerland; \\ ${ }^{10}$ Department of Pulmonary Medicine, Graduate School of Medical Science, Kyoto Prefectural University of Medicine, Kyoto, Japan; ${ }^{11}$ Bannan \\ Central Hospital, Iwata, Shizuoka, Japan; ${ }^{12}$ Department of Oncology, Montefiore Medical Center/Albert Einstein College of Medicine, Bronx, NY, \\ USA; ${ }^{13}$ Department of Thoracic Surgery, Roswell Park Comprehensive Cancer Center, Buffalo, NY, USA; ${ }^{14}$ Department of Thoracic Surgery, Kaiser \\ Permanente Oakland Medical Center, Kaiser Permanente Northern California, Oakland, CA, USA \\ Contributions: (I) Conception and design: M Li; (II) Administrative support: M Li; (III) Provision of study materials or patients: M Li; (IV) Collection \\ and assembly of data: J Jiang, Y Wang, Y Gao; (V) Data analysis and interpretation: J Jiang, M Li; (VI) Manuscript writing: All authors; (VII) Final \\ approval of manuscript: All authors. \\ Correspondence to: Min Li. Department of Respiratory Medicine, Xiangya Hospital, Central South University, Changsha, China. \\ Email: limin2050@csu.edu.cn.
}

Background: In recent years, a series of clinical trials have explored the application of neoadjuvant immunotherapy or chemoimmunotherapy in non-small cell lung cancer (NSCLC). However, no randomized control trials comparing neoadjuvant immunotherapy with chemoimmunotherapy have yet been reported. This study aimed to summarize and compare the efficacy and safety of neoadjuvant immunotherapy and chemoimmunotherapy in NSCLC.

Methods: Literature focusing on the efficacy and safety of neoadjuvant immunotherapy or chemoimmunotherapy in NSCLC published before June 2021 was retrieved from PubMed, Embase, and the Cochrane Library. Study endpoints included major pathological response (MPR), complete pathological response (pCR), treatment-related adverse events (TRAEs), severe adverse events (SAEs), resection rate, surgical delay rate, and conversion to thoracotomy. The risk of bias was assessed using the Cochrane bias risk assessment tool. Subgroup and sensitivity analyses were further performed.

Results: A total of 988 patients from 16 studies were included in this meta-analysis. For patients who received neoadjuvant immunotherapy with single/combined ICIs or chemoimmunotherapy, the pooled MPR rate was $43.5 \%$ and the pooled pCR rate was $21.9 \%$. The pooled incidence of TRAEs and SAEs were $54.8 \%$ and $15.3 \%$, respectively. The pooled resection rate was $85.8 \%$, the surgical delay rate was $7.4 \%$, and the conversion rate was $17.4 \%$. Patients who received neoadjuvant chemoimmunotherapy had remarkably improved pathological response (MPR rate: $53.3 \%$ vs. $28.6 \%$; pCR rate: $28.6 \%$ vs. $9.9 \%$ ) compared with those receiving neoadjuvant single-agent immunotherapy, while the incidence of SAEs (18.0\% vs. $12.3 \%)$ and surgical delay rate $(3.8 \%$ vs. $7.4 \%)$ did not significantly increase. Neoadjuvant nivolumab combined with ipilimumab also achieved a high pCR rate $(28.6 \%)$ with tolerable toxicity. Nivolumab- and pembrolizumabbased neoadjuvant therapy showed a higher MPR rate (nivolumab 51.5\%, pembrolizumab 46.8\%) and pCR rate (nivolumab 29.1\%, pembrolizumab 31.5\%). Besides, patients with positive programmed death-ligand 1 (PD-L1) expression [tumor proportion score (TPS) $\geq 1 \%$ ] exhibited favorable pathological responses than 
PD-L1 negative patients.

Discussion: Overall, neoadjuvant immunotherapy or chemoimmunotherapy is effective and safe in NSCLC. Compared with single-agent immunotherapy, neoadjuvant chemoimmunotherapy provides a significant improvement in pathological response without increasing the incidence of SAEs or surgical delay. These results need further confirmation by more large-scale randomized controlled trials.

Keywords: Non-small cell lung cancer (NSCLC); neoadjuvant immunotherapy; neoadjuvant chemoimmunotherapy; immune checkpoint inhibitors (ICIs); efficacy and safety

Submitted Nov 19, 2021. Accepted for publication Feb 18, 2022.

doi: $10.21037 /$ tlcr-22-75

View this article at: https://dx.doi.org/10.21037/tlcr-22-75

\section{Introduction}

According to the 2020 global cancer statistics, non-small cell lung cancer (NSCLC) accounts for $80-85 \%$ of all lung cancers worldwide and remains the leading cause of cancer deaths (1). Approximately $70 \%$ of NSCLC patients are diagnosed at an advanced stage, leading to a 5-year survival rate less than $18 \%$ even after comprehensive treatment (2). Over the past decades, the early detection of NSCLC has gradually increased with the wider adoption of chest lowdose CT as a screening modality (3). For patients diagnosed with stages I, II, and selected stage III NSCLC, surgical resection with curative intent is considered to be the best treatment option. However, only $20-25 \%$ of tumors overall are suitable for potential curative resection. Moreover, these patients have a high risk of postoperative recurrence, ranging from $25 \%$ to $70 \%$ based on the disease stage (4).

Neoadjuvant therapy, defined as systemic anticancer treatment given before surgery, is an accepted practice in NSCLC (5). Compared with adjuvant therapy, neoadjuvant therapy has potential advantages including early treatment of micrometastasis, reducing tumor burden before surgery, and better tolerability $(6,7)$. Pathological remission, including major pathological response (MPR) and complete pathological response (pCR), is currently used as a surrogate endpoint to predict survival benefit in clinical trials focusing on neoadjuvant chemotherapy (8). A meta-analysis conducted by the NSCLC Meta-analysis Collaborative Group involving 15 randomized clinical trials in patients with stages IB-IIIA disease has demonstrated that compared with surgery alone, preoperative chemotherapy significantly improves overall survival, time to distant recurrence, and recurrence-free survival in resectable NSCLC (9). However, the median rate of pCR from 15 trials of neoadjuvant chemotherapy was only $4 \%$ (range, $0-16 \%$ ), which suggests that more effective neoadjuvant therapy modalities are required to further improve prognosis (10).

Immunotherapy with immune checkpoint inhibitors (ICIs), which boost antitumor immunity by blocking inhibitory signaling through checkpoint receptors expressed on $\mathrm{T}$ lymphocytes and their ligands expressed in tumor cells, has revolutionized the treatment of various cancers, including NSCLC $(4,11)$. In recent years, the use of ICIs for the treatment of NSCLC has considerably increased. Monoclonal antibodies that target the programmed cell death protein 1/programmed death-ligand 1 (PD-1/PD-L1) axis have been approved as first- and second-line treatments for advanced NSCLC worldwide (12). So far, several clinical trials have reported the efficacy and safety of neoadjuvant immunotherapy (13-18) or chemoimmunotherapy (19-28) in stages I-III NSCLC. Investigational ICIs included PD-1/ PD-L1 inhibitors such as nivolumab $(13,18,25,27,28)$, pembrolizumab (16,20), sintilimab (15), durvalumab (26), atezolizumab (14,19), avelumab (23), and a CTLA-4 inhibitor ipilimumab (24). In NSCLC patients who receive preoperative immunotherapy, the naive tumor can serve as a "vaccine" boosting the activation of T lymphocytes (29). Based on available research data from previous studies, patients who received neoadjuvant immunotherapy or chemoimmunotherapy before surgery had improved pathological response and downstaging of the tumor compared to those who received neoadjuvant chemotherapy. However, most of these studies are single-armed trials with small sample sizes. On the other hand, while neoadjuvant immunotherapy or chemoimmunotherapy offers numerous advantages, immune-related adverse events (irAEs) have attracted significant attention (30,31). The occurrence of severe irAEs can lead to delayed surgical resection or even death. Moreover, increasing complexity of the surgical 
field in the chest, which is caused by fibrosis and nodal flares, has been observed in patients receiving neoadjuvant immunotherapy, even with tumor downstaging (13). So far, no data from randomized controlled trials on neoadjuvant immunotherapy and chemoimmunotherapy have been reported. Therefore, in order to improve the knowledge of and compare the clinical benefits between neoadjuvant immunotherapy and chemoimmunotherapy in NSCLC, a meta-analysis on their efficacy and safety, based on current data from clinical trials, is necessary.

Herein, we conducted a systematic review and metaanalysis of clinical trials focusing on neoadjuvant immunotherapy or chemoimmunotherapy in NSCLC. We analyzed efficacy- and safety-related endpoints including MPR, pCR, incidence of treatment-related adverse events (TRAEs) and severe adverse events (SAEs), resection rate, surgical delay rate, and conversion rate in resectable stages I-III NSCLC patients who received neoadjuvant immunotherapy or chemoimmunotherapy. We further compared these endpoints among different treatment modes and ICI types, and summarized the profiles of TRAEs and SAEs. This meta-analysis was conducted and reported in accordance with the PRISMA reporting checklist (32) (available at https://tlcr.amegroups.com/ article/view/10.21037/tlcr-22-75/rc).

\section{Methods}

\section{Literature search strategy and study selection}

It was registered in PROSPERO with the registration number CRD42021262202. We combined the English search terms ("NSCLC" or "carcinoma, non-small cell lung" or "nonsmall cell lung cancer") and ("neoadjuvant immunotherapy" or "neoadjuvant chemoimmunotherapy" or "neoadjuvant immune checkpoint inhibitor") and ("surgery" or "resection" or "lobectomy") in PubMed, Embase, and the Cochrane Library to identify articles published before 30 June 2021. At the same time, we also searched the unpublished updated data of ongoing clinical trials of neoadjuvant immunotherapy or chemoimmunotherapy in NSCLC from international congresses such as ASCO, AACR, ESMO, and other congresses up to 30 June 2021. All publications were limited to human subjects and English language.

Inclusion criteria of publications were defined according to the PICOS criteria, which was listed as follows: (I) patients: resectable stages I-III NSCLC which was histologically confirmed in tissue; (II) intervention: neoadjuvant ICIs, including PD-1/PD-L1 inhibitors and CTLA-4 inhibitors, either combined with chemotherapy or not; (III) comparator: how effective and safe are the different neoadjuvant treatment regimens; (IV) outcomes: MPR, pCR, incidence of TRAEs, incidence of SAEs, resection rate, surgical delay rate, and conversion to thoracotomy; (V) study design: randomized controlled trials, non-randomized controlled trials, prospective cohort studies. Publications were excluded if they met any of the following criteria: (I) retrospective studies; (II) antiNSCLC treatment was performed before neoadjuvant therapy; (III) studies did not focus on any of the endpoints, including MPR, pCR, incidence of TRAEs, incidence of SAEs, resection rate, surgical delay rate, or conversion rate; (IV) publications written in any other language than English; (V) the number of enrolled patients was not more than 10; (VI) repeated publications, case reports, comments, expert opinion, and reviews; (VII) violation of any of the above inclusion criteria. Subsequently, the full texts of the relevant articles and conference abstracts were retrieved to assess eligibility. References to relevant reports were also reviewed manually to identify other studies. Two reviewers (JJ, YW) independently screened titles and abstracts using the above-mentioned search strategies to identify and assess potentially eligible studies. Publications from the reference lists of retrieved articles were also searched. The full-texts of all potentially relevant trials and conference abstracts were assessed using predefined eligibility criteria. Disagreements were resolved by discussion with a third researcher (ML).

\section{Assessment of the risk of bias}

According to the Cochrane Handbook for Systematic Reviews of Interventions, the risk of bias of randomized trials was assessed by the Cochrane risk-of-bias tool, and that of non-randomized studies was assessed by the ROBINS-I tool. The risk of bias of randomized studies included selection bias (including random sequence generation and allocation concealment), performance bias (blinding of participants and personnel), detection bias (blinding of outcome assessment), attrition bias (incomplete outcome data), reporting bias (selective reporting), and other biases. Non-randomized studies were assessed using the ROBINS-I tool with respect to the following categories: confounding, selection, intervention classification, deviation from intervention, missing data, measurement of outcome, and selection of reported result. 


\section{Publication bias}

Funnel plot, Egger's test and Begg's test were used to assess the publication bias of included studies.

\section{Data extraction}

Two researchers (JJ and YW) independently extracted relevant data using a pre-designed form. Disputed data were discussed with a third researcher (ML). The extracted information were as follows: (I) study features, including the first author, year of publication, basic study design, main inclusion criteria of patients, neoadjuvant treatment regimens, and sample size; (II) baseline characteristics of the enrolled patients, including gender, age, and proportion of squamous cell carcinoma; (III) data on endpoints, including MPR, pCR, incidence of TRAEs, incidence of SAEs, patients with resection, surgical delay rate, and conversion to thoracotomy. To ensure the homogeneity of different studies, the MPR rate, pCR rate, resection rate, surgical delay rate, and conversion rate were all defined as the ratio to the total number of the intention-to-treat population for meta-analysis. The extracted data were mostly represented by counts and percentages.

\section{Definitions of endpoints}

The pathological responses of patients, including MPR and pCR, were used to evaluate the efficacy of neoadjuvant immunotherapy or chemoimmunotherapy. MPR was defined as no more than $10 \%$ viable tumor cells remaining in tumors and lymph nodes on postoperative pathological review, and pCR was defined as no viable tumor cells remaining on postoperative pathological review. The safety-related endpoints included neoadjuvant TRAEs, SAEs, resection rate, surgical delay rate, and conversion to thoracotomy. TRAEs and SAEs were evaluated according to the Common Terminology Criteria for Adverse Events (CTCAE). SAEs were defined as grade 3-5 TRAEs. The resection rate referred to the ratio of patients who underwent surgical resection to the intention-to-treat population. The surgical delay rate was defined as the ratio of patients with surgical delay caused by TRAEs to the intention-to-treat population. The conversion rate referred to the ratio of patients scheduled to undergo videoassisted thoracoscopic surgery (VATS) or robotic approach but converted to thoracotomy during the operation due to surgical difficulty, complexity, and/or unexpected complications.

\section{Statistical analysis}

Data analysis was performed using Review Manager Software (version 5.4). Since most of the included studies were single-arm clinical trials, we performed metaanalysis with non-comparative binary data of MPR, pCR, adverse events, resection, and conversion in Review Manager Software, as described in a previous study (33). Briefly, the $\log [$ odds ratio] of an endpoint and its standard error (SE) were calculated with the following formula: $\log [$ odds ratio $]=\ln (X /(n-x)) ; S E=\sqrt{1 / X+1 /(n-x)} ;$ where $\mathrm{X}$ refers to the number of events, and $n$ refers to the total number of patients. Then, $\log$ [odds ratio] and SE were input into the Review Manager Software for analysis. Odds ratio $(\mathrm{OR})$ and $95 \%$ confidence interval $(\mathrm{CI})$ were the effect measures. Finally, the pooled prevalence $(\mathrm{P})$ of an endpoint and its $95 \%$ CI were calculated with the following formula: $\mathrm{P}=\mathrm{OR} /(1+\mathrm{OR})$; lower limit $(\mathrm{LL})$ of $95 \% \mathrm{CI}=$ $\mathrm{LL}_{\mathrm{OR}} /\left(1+\mathrm{LL}_{\mathrm{OR}}\right)$; upper limit $(\mathrm{UL})$ of $95 \% \mathrm{CI}=\mathrm{UL}_{\mathrm{OR}} /(1$ $\left.+\mathrm{UL}_{\mathrm{OR}}\right)$. The $\chi^{2}$ test and $\mathrm{I}^{2}$ test were used to evaluate the heterogeneity of the enrolled studies. If the heterogeneity was significant, the random effects model was adopted for meta-analysis; otherwise, the fixed effects model was used. We further performed subgroup analysis and sensitivity analysis to explore the sources of heterogeneity. For subgroup analysis based on PD-L1 expression, histology and smoking status, available individual data was extracted from each study, and the Mantel-Haenszel random effect method was used to obtain the pooled OR and the corresponding 95\% CI. A sensitivity analysis was performed by removing one study at a time to evaluate whether the results could be significantly affected by a single study. Significance was fixed at $\mathrm{P}<0.05$ (two-tailed).

\section{Results}

\section{Literature search results}

The literature search and study selection process are shown in Figure 1. The search strategy identified a total of 663 records. After removing duplicate records, the titles and abstracts of 344 records were screened, and 312 records were excluded as they did not meet the inclusion criteria. Full-text articles or conference abstracts of 32 studies were read in detail and further assessed for eligibility. Finally, a total of 16 studies and 988 NSCLC patients were enrolled for quantitative meta-analysis. Study characteristics were shown based on the categories of neoadjuvant treatment regimens (Table 1). Among these studies, 14 were single-arm 


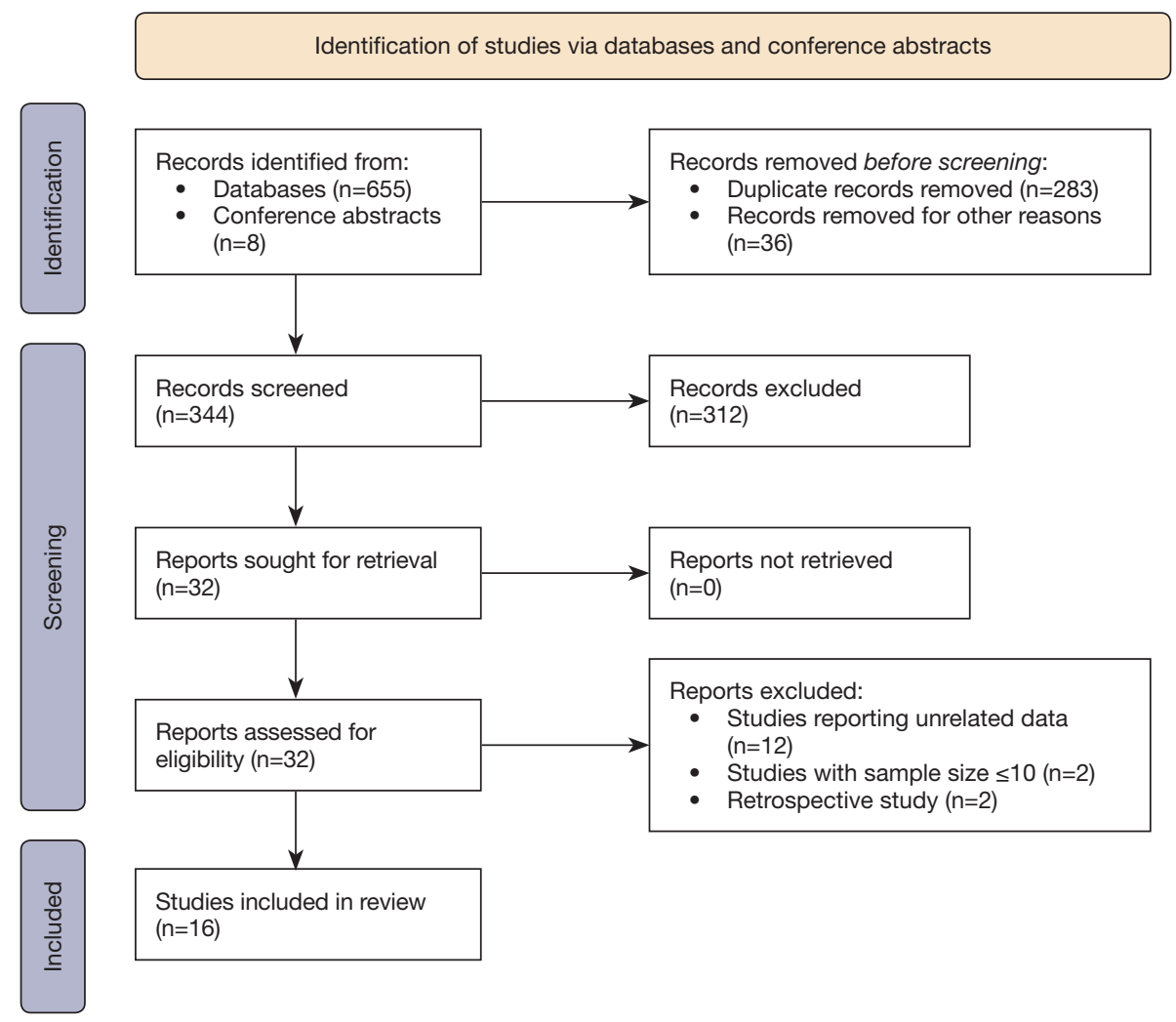

Figure 1 Flow chart of literature search and study selection.

open-label cohort studies, and the other 2 were dual-arm openlabel randomized trials. The Provencio 2020 (28), Rothschild 2021 (26), and Wang 2021 (21) studies enrolled patients with stage III NSCLC. Six studies focused on neoadjuvant immunotherapy alone (including 1 study on dual ICIs), while the other 10 explored the efficacy and safety of neoadjuvant chemoimmunotherapy. Investigational ICIs included nivolumab, pembrolizumab, atezolizumab, durvalumab, sintilimab, ipilimumab, and avelumab. The risk of bias of randomized trials and non-randomized studies were assessed using the Cochrane risk-of-bias tool and ROBINS-I tool, respectively, which was displayed in Figure S1. Publication bias of included studies was overall limited, as suggested by funnel plots (Figure S2).

\section{Pooled analysis of efficacy-and safety-related endpoints}

Research data on efficacy- and safety-related endpoints in these clinical trials are shown in Table 2. We firstly conducted a meta-analysis of the MPR rate, pCR rate, incidence of
TRAEs and SAEs, resection rate, surgical delay rate, and conversion rate for all studies (Figure 2 and Table 3). The pooled MPR rate was $43.5 \%$ (95\% CI: $32.4-55.0 \%$ ) and the pooled pCR rate was $21.9 \%$ (95\% CI: $15.3-30.6 \%)$. There was significant heterogeneity for both MPR $(\mathrm{P}<0.001$, $\left.\mathrm{I}^{2}=85 \%\right)$ and $\mathrm{pCR}\left(\mathrm{P}<0.001, \mathrm{I}^{2}=81 \%\right)$, so the random effects model was adopted. The pooled incidence of TRAEs and SAEs were $54.8 \%$ (95\% CI: $41.5-67.3 \%$ ) and $15.3 \%$ (95\% CI: $10.7-21.3 \%)$, respectively. The pooled resection rate was $85.8 \%$ (95\% CI: 80.4-89.8\%) and the pooled surgical delay rate was $7.4 \%$ (95\% CI: $3.8-12.3 \%$ ). Only 3 studies reported the incidence of conversion to thoracotomy. The pooled conversion rate based on these studies was $17.4 \%$ (95\% CI: 7.4-35.9\%). The random effects model was used for meta-analysis of the above endpoints due to significant heterogeneity among the included studies. It is worth mentioning that only patients who received neoadjuvant ICIs were included in the pooled analysis of these endpoints. Therefore, those who received neoadjuvant chemotherapy alone in the CheckMate 816 trial were excluded herein. 


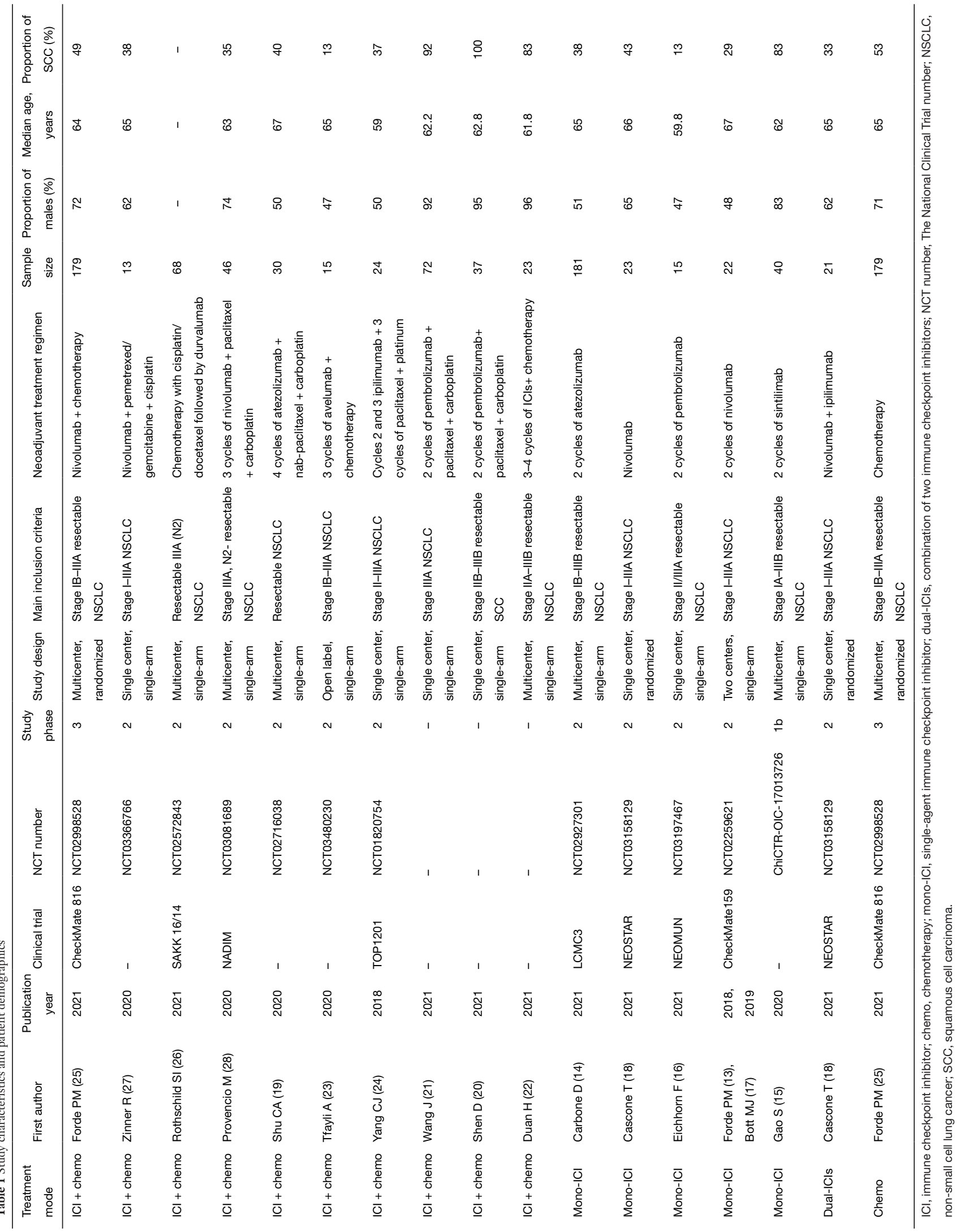




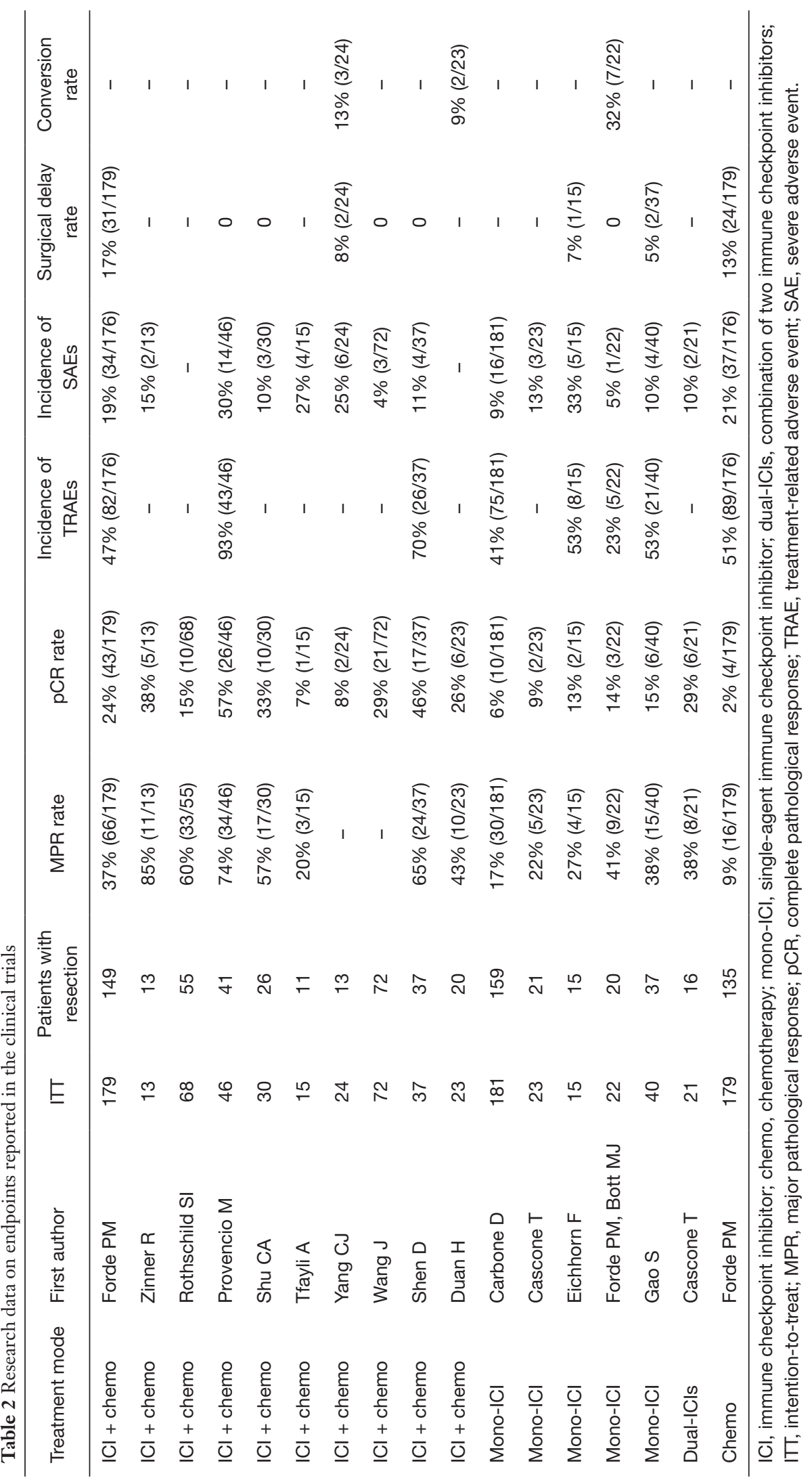



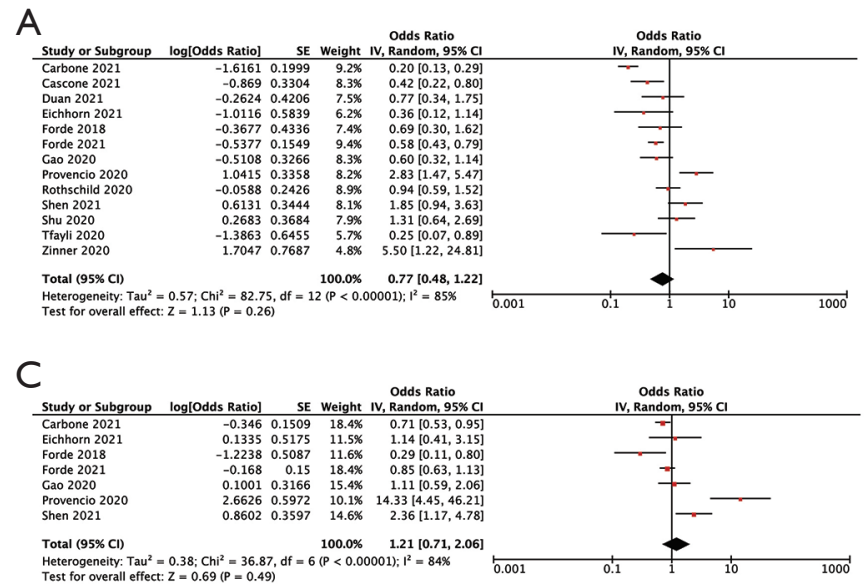

E

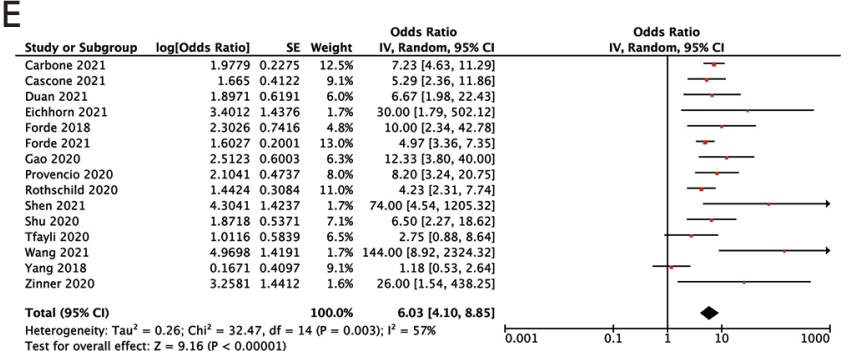

B

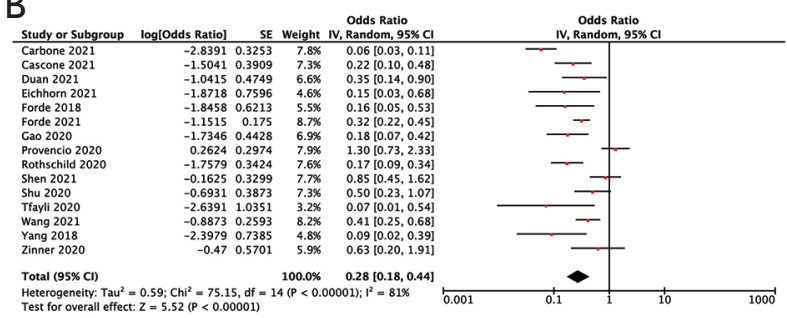

D

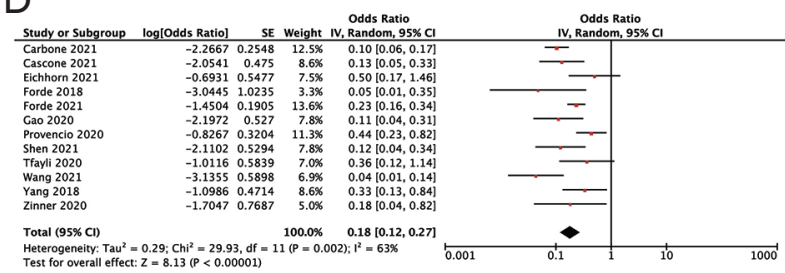

$\mathrm{F}$

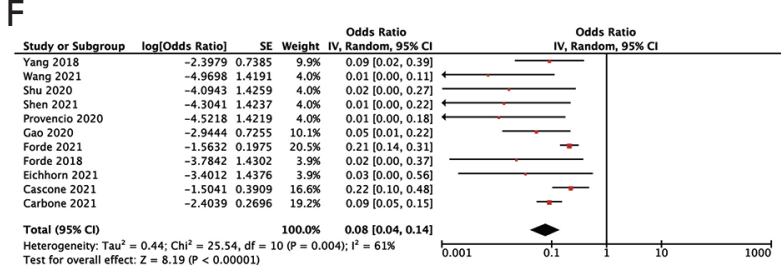

G

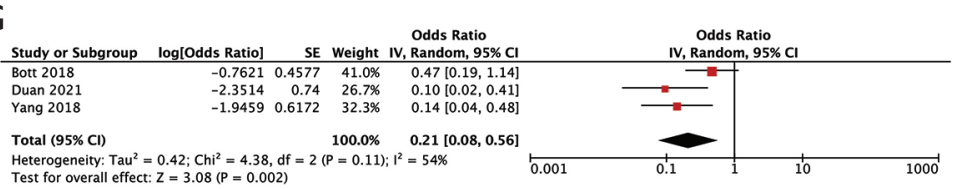

Figure 2 Forest plot of the efficacy and safety of neoadjuvant immunotherapy and chemoimmunotherapy. (A) MPR rate; (B) pCR rate; (C) incidence of TRAEs; (D) Incidence of SAEs; (E) resection rate; (F) surgical delay rate; (G) conversion rate. MPR, major pathological response; pCR, complete pathological response; TRAE, treatment-related adverse event; SAE, severe adverse event; SE, standard error; IV, inverse variance; $\mathrm{CI}$, confidence interval.

\section{Exploratory subgroup analysis}

\section{Neoadjuvant treatment modes}

To identify the potential sources of heterogeneity, exploratory subgroup analysis was performed. Among all the included studies, there were 4 kinds of neoadjuvant treatment modes used in patients with resectable NSCLC, including ICI plus chemotherapy, mono-ICI, dual ICIs (nivolumab plus ipilimumab), and chemotherapy alone. Subgroup analysis based on neoadjuvant treatment modes is shown in Figure 3 and Table 3. The pooled MPR rates for ICI plus chemotherapy, mono-ICI, dual ICIs, and chemotherapy alone were $53.3 \%$ (95\% CI: 40.5-65.8\%), $28.6 \%$ (95\% CI: $18.7-40.8 \%$ ), $38.3 \%$ (95\% CI: $20.6-$ $59.7 \%$ ), and $9.1 \%$ (95\% CI: $5.7-13.8 \%$ ), respectively. A significant difference was observed among these subgroups
$(\mathrm{P}<0.001)$ (Figure $3 A)$. The pooled $\mathrm{pCR}$ rates for ICI plus chemotherapy, mono-ICI, dual ICIs, and chemotherapy alone were $28.6 \%$ (95\% CI: $20.0-38.7 \%$ ), $9.9 \%$ (95\% CI: $5.7-15.3 \%$ ), $28.6 \%$ (95\% CI: $13.8-50.7 \%$ ), and $2.0 \%$ (95\% CI: $1.0-5.7 \%)$, respectively. The difference in the pCR rate among subgroups was also statistically significant $(\mathrm{P}<0.001)$ (Figure 3B). These results highlight the advantages of neoadjuvant chemoimmunotherapy and dual ICIs over other treatment modes in terms of pathological response.

The incidence of TRAEs in patients who received neoadjuvant chemoimmunotherapy tended to be higher [73.9\% (95\% CI: 43.2-91.3\%)] compared with the monoICI [42.9\% (95\% CI: 32.4-53.9\%)] and chemotherapy alone [49.7\% (95\% CI: 42.5-57.1\%)] subgroups (Figure $3 C)$. However, no significant difference was found $(\mathrm{P}=0.15)$. 
Table 3 Comparison of endpoints among different subgroups after neoadjuvant immunotherapy

\begin{tabular}{|c|c|c|c|c|c|c|c|}
\hline Subgroups & MPR rate $(95 \% \mathrm{Cl})$ & pCR rate $(95 \% \mathrm{Cl})$ & $\begin{array}{c}\text { Incidence of } \\
\text { TRAEs (95\% Cl) }\end{array}$ & $\begin{array}{c}\text { Incidence of } \\
\text { SAEs }(95 \% \mathrm{Cl})\end{array}$ & $\begin{array}{c}\text { Resection rate } \\
\quad(95 \% \mathrm{Cl})\end{array}$ & $\begin{array}{l}\text { Surgical delay } \\
\text { rate }(95 \% \mathrm{Cl})\end{array}$ & $\begin{array}{c}\text { Conversion rate } \\
\qquad(95 \% \mathrm{Cl})\end{array}$ \\
\hline Pooled & $43.5(32.4-55.0)$ & $21.9(15.3-30.6)$ & $54.8(41.5-67.3)$ & $15.3(10.7-21.3)$ & $85.8(80.4-89.8)$ & $7.4(3.8-12.3)$ & $17.4(7.4-35.9)$ \\
\hline $\mathrm{ICI}+$ chemo & $53.3(40.5-65.8)$ & $28.6(20.0-38.7)$ & $73.9(43.2-91.3)$ & $18.0(12.3-26.5)$ & $84.4(75.8-90.3)$ & $3.8(1.0-13.0)$ & - \\
\hline Mono-ICl & $28.6(18.7-40.8)$ & $9.9(5.7-15.3)$ & $42.9(32.4-53.9)$ & $12.3(6.5-21.3)$ & $89.2(84.9-92.4)$ & $7.4(4.8-11.5)$ & - \\
\hline Chemo & $9.1(5.7-13.8)$ & $2.0(1.0-5.7)$ & $49.7(42.5-57.1)$ & $20.6(15.3-27.0)$ & $75.4(68.6-81.2)$ & $7.4(4.8-12.3)$ & - \\
\hline$P$ value & $<0.001$ & $<0.001$ & 0.15 & 0.32 & 0.002 & 0.02 & - \\
\hline \multicolumn{8}{|l|}{$\mathrm{ICl}$ types } \\
\hline Nivolumab & $51.5(32.4-70.0)$ & $29.1(16.0-46.2)$ & $60.0(22.5-88.5)$ & $18.7(12.3-27.5)$ & $84.9(80.3-88.6)$ & $12.3(6.5-23.1)$ & - \\
\hline Atezolizumab & 33.3 (7.4-75.9) & $14.5(2.0-58.2)$ & $41.5(34.6-48.7)$ & $9.1(5.7-14.5)$ & $87.7(82.5-91.5)$ & $6.5(2.0-18.7)$ & - \\
\hline Sintilimab & $37.5(24.2-53.3)$ & $15.3(6.5-29.6)$ & $52.6(37.1-67.3)$ & $9.9(3.8-23.7)$ & $92.5(79.2-97.6)$ & $4.8(1.0-18.0)$ & - \\
\hline Avelumab & $20.0(6.5-47.1)$ & $6.5(1.0-35.1)$ & - & $26.5(10.7-53.3)$ & $73.3(46.8-89.6)$ & - & - \\
\hline$P$ value & 0.39 & 0.18 & 0.06 & 0.11 & 0.22 & 0.07 & - \\
\hline
\end{tabular}

$\mathrm{ICl}$, immune checkpoint inhibitor; chemo, chemotherapy; mono-ICl, single-agent immune checkpoint inhibitor; dual-ICls, combination of two immune checkpoint inhibitors; MPR, major pathological response; pCR, complete pathological response; TRAE, treatment-related adverse event; SAE, severe adverse event; $\mathrm{Cl}$, confidence interval.

No data on the incidence of TRAEs was provided in the Cascone 2021 study (18), the only one study using dual ICIs. The incidence of SAEs in the ICI plus chemotherapy, mono-ICI, dual ICIs, and chemotherapy alone subgroups were $18.0 \%$ (95\% CI: $12.3-26.5 \%$ ), $12.3 \%$ (95\% CI: 6.5-21.3\%), 9.9\% (95\% CI: $2.0-31.0 \%$ ), and $20.6 \%$ (95\% CI: $15.3-27.0 \%)$, respectively. There was no significant difference among subgroups $(\mathrm{P}=0.32)$. The resection rates for the ICI plus chemotherapy, mono-ICI, dual ICIs, and chemotherapy alone subgroups were $84.4 \%$ (95\% CI: $75.8-90.3 \%$ ), 89.2\% (95\% CI: 84.9-92.4\%), 76.2\% (95\% CI: $53.9-89.7 \%$ ), and 75.4\% (95\% CI: 68.6-81.2\%), respectively. Patients who received neoadjuvant ICI plus chemotherapy had the lowest surgical delay rate [3.8\% (95\% CI: $1.0-13.0 \%)$ ]. Since only 3 studies reported the data of conversion rate, subgroup analysis on this endpoint was not conducted. Through this subgroup analysis, heterogeneity among studies of the mono-ICI subgroup was remarkably decreased. Significant heterogeneity was still observed for the MPR rate of the mono-ICI subgroup and all endpoints of the ICI plus chemotherapy subgroup.

\section{ICI types}

Different types of ICIs used in the included studies could be a source of heterogeneity. Therefore, we conducted a subgroup analysis of ICI types to explore the potential correlations between ICI types and endpoints (Figure 4). As shown in Table 3, nivolumab- and pembrolizumab-based neoadjuvant therapy showed a higher MPR rate $[51.5 \%$ (95\% CI: $32.4-70.0 \%$ ) for nivolumab, $46.8 \%$ (95\% CI: $15.3-81.1 \%$ ) for pembrolizumab] and pCR rate $[29.1 \%$ (95\% CI: $16.0-46.2 \%$ ) for nivolumab, 31.5\% (95\% CI: $18.0-49.2 \%$ ) for pembrolizumab], while avelumab-based neoadjuvant therapy had a relatively lower MPR rate [20.0\% (95\% CI: $6.5-47.1 \%)]$ and pCR rate [6.5\% (95\% CI: $1.0-35.1 \%)]$. However, no significant differences were observed among these subgroups. The pooled incidence of TRAEs and SAEs for atezolizumab- and sintilimabbased neoadjuvant therapy appeared to be lower than other ICIs, without statistical significance. The resection rates of different ICIs were overall comparable. Patients who received pembrolizumab-based neoadjuvant therapy tended to have less surgical delay [1.0\% (95\% CI: 0.0-6.5\%)], while 
A

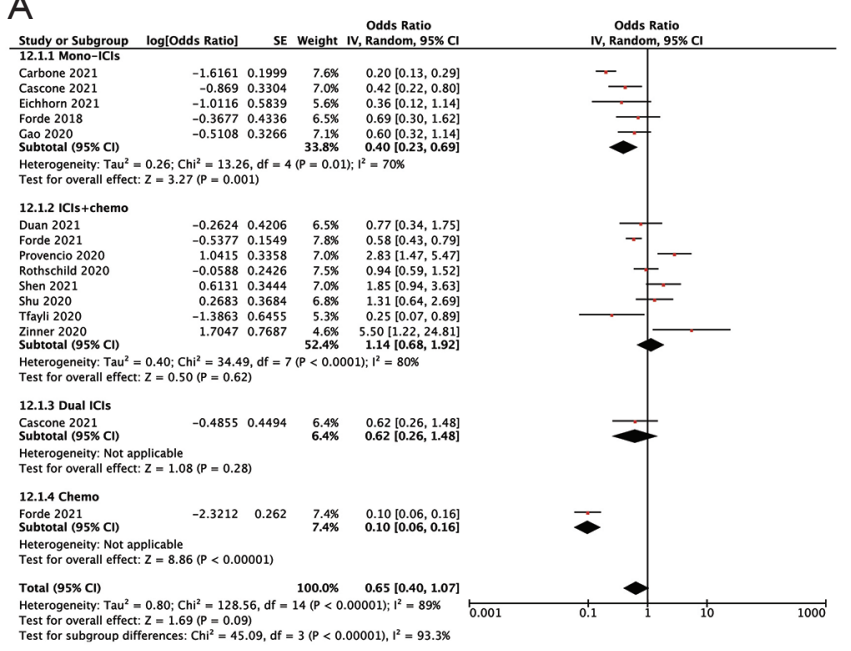

C

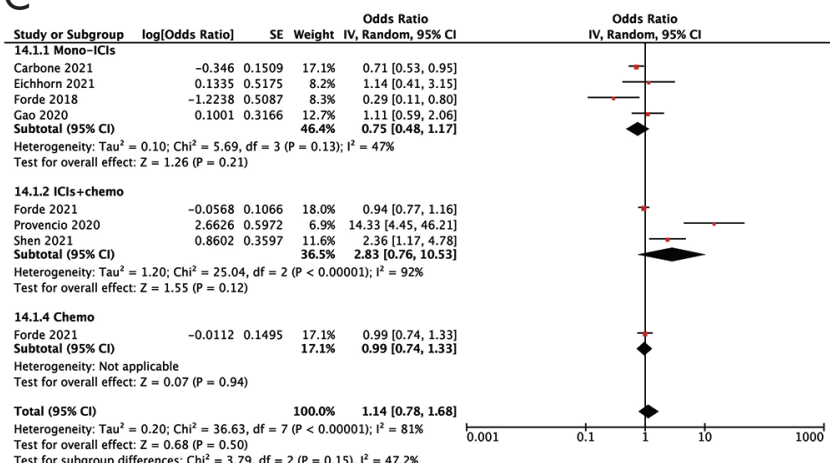

E

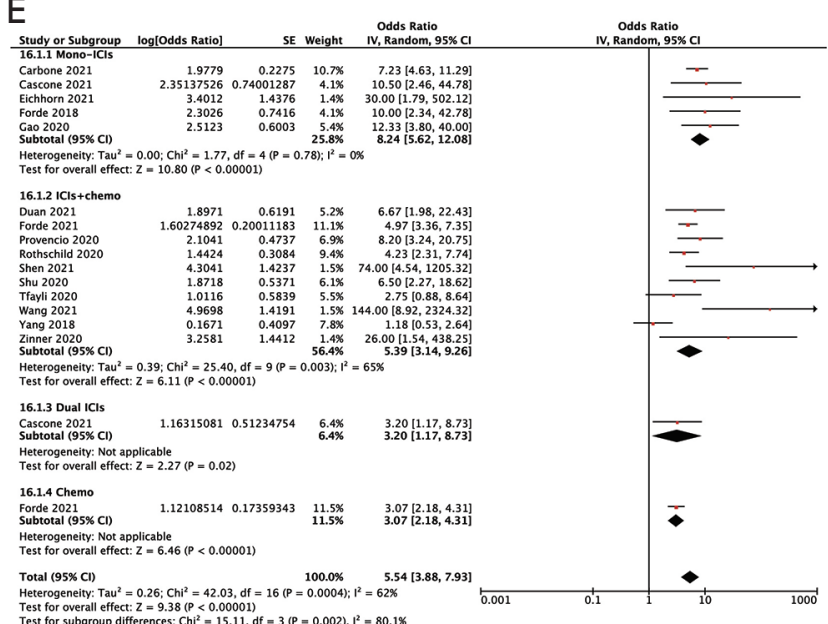

B

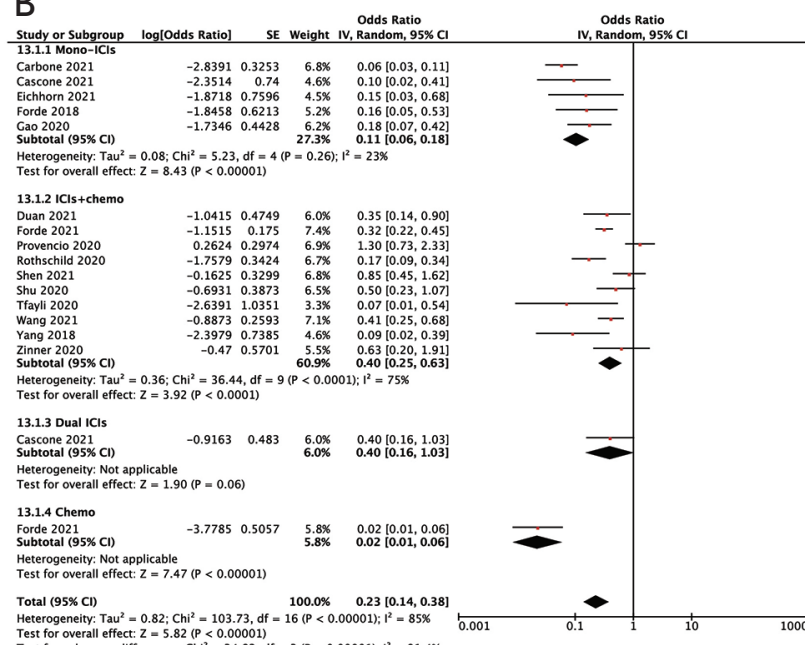

Test for overall effect. $Z=5.82(P<0.00001)$
Test for subgroup differences: $\mathrm{Chi}^{2}=34.82$, df $=3(\mathrm{P}<0.00001), \mathrm{I}^{2}=91.4 \%$

D

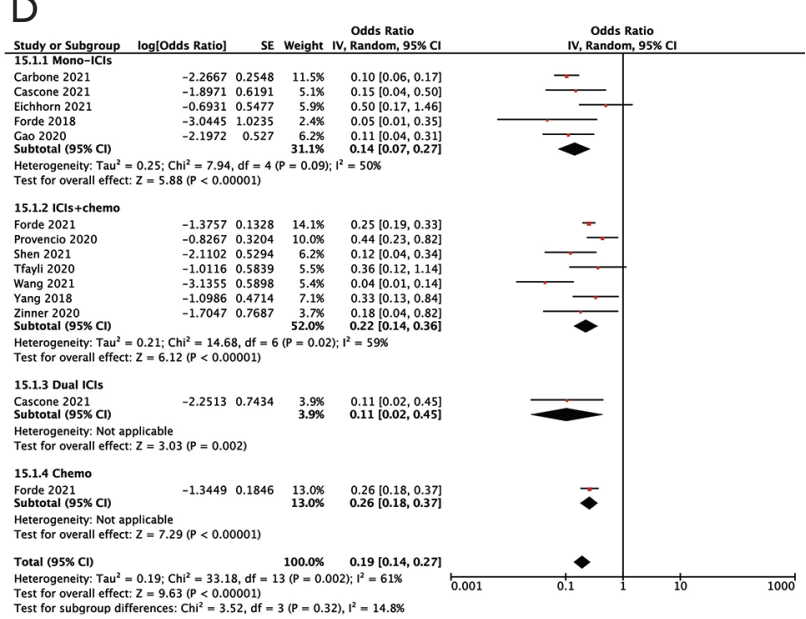

$\mathrm{F}$

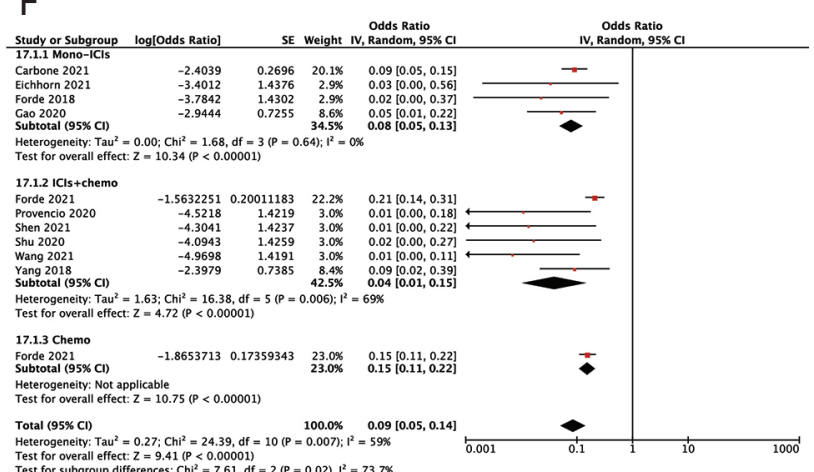

Figure 3 Forest plot of the efficacy and safety of subgroup analysis based on different neoadjuvant treatment modes. (A) MPR rate; (B) pCR rate; (C) incidence of TRAEs; (D) incidence of SAEs; (E) resection rate; (F) surgical delay rate. MPR, major pathological response; pCR, complete pathological response; TRAE, treatment-related adverse event; SAE, severe adverse event; ICIs, immune checkpoint inhibitors; chemo, chemotherapy; ICI + chemo, immune checkpoint inhibitors plus chemotherapy; SE, standard error; IV, inverse variance; CI, confidence interval. 


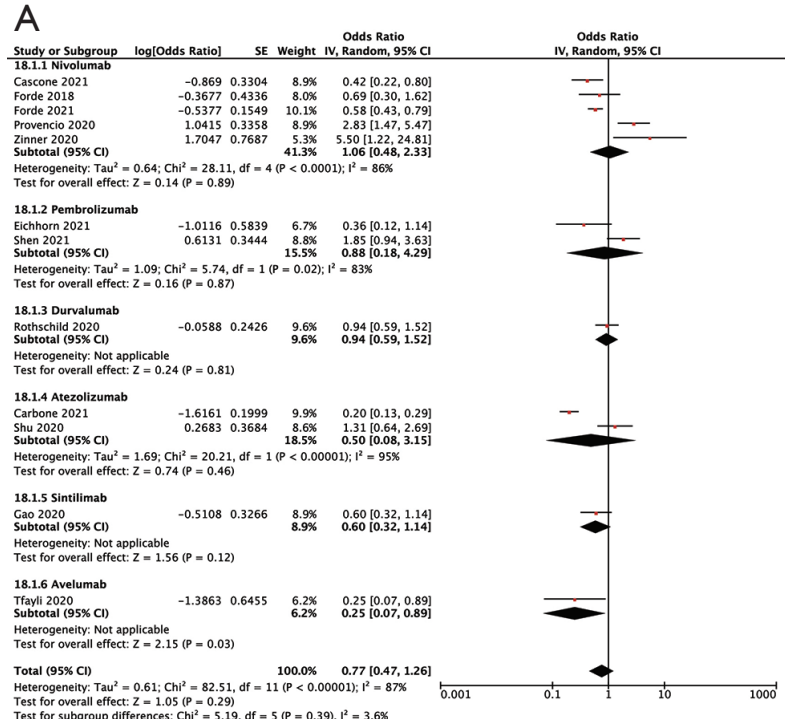

C

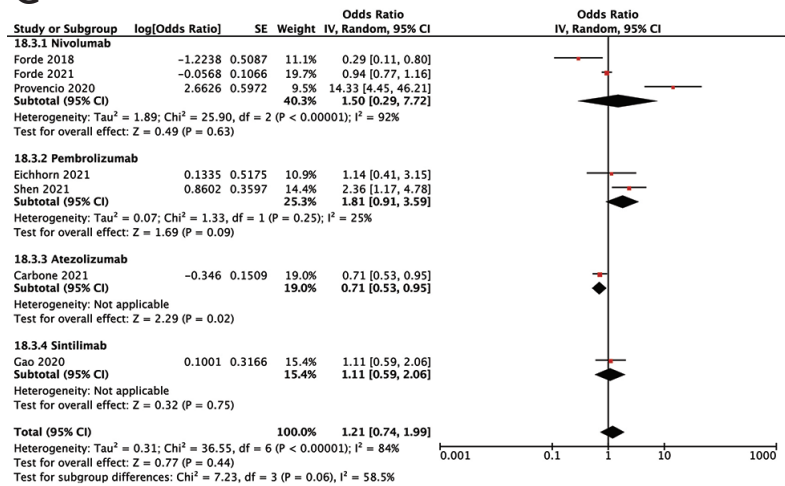

$\mathrm{E}$

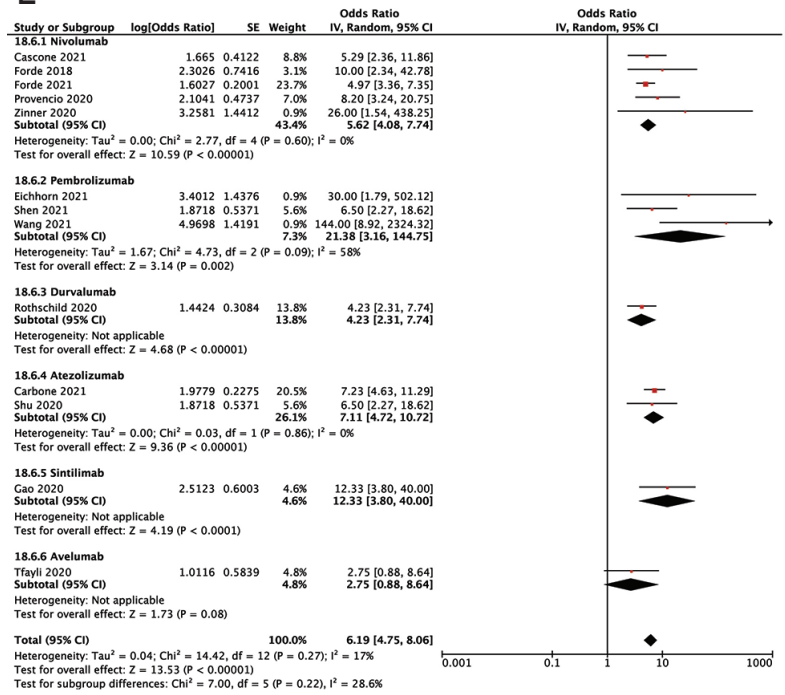

B

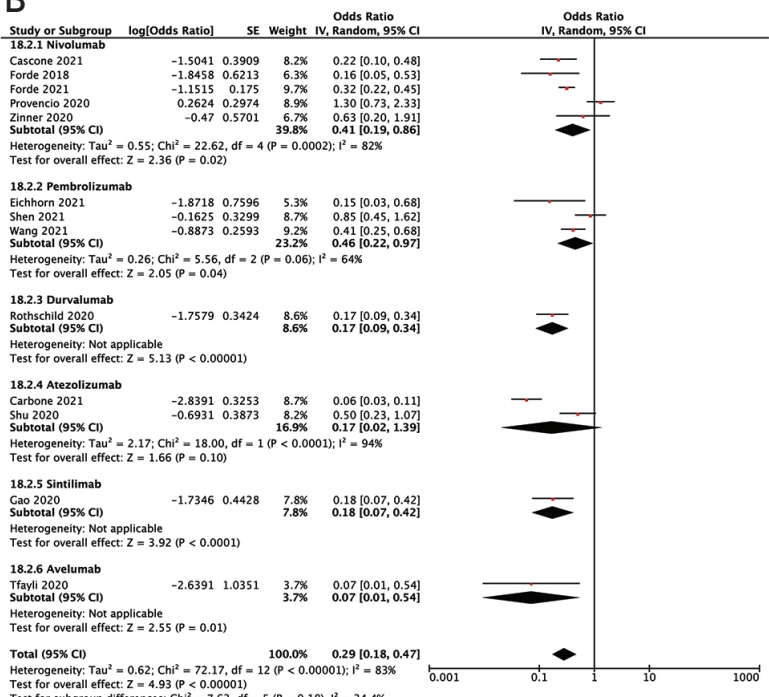

Test for overal effect: $Z=4.93(P<0.00001)$

D
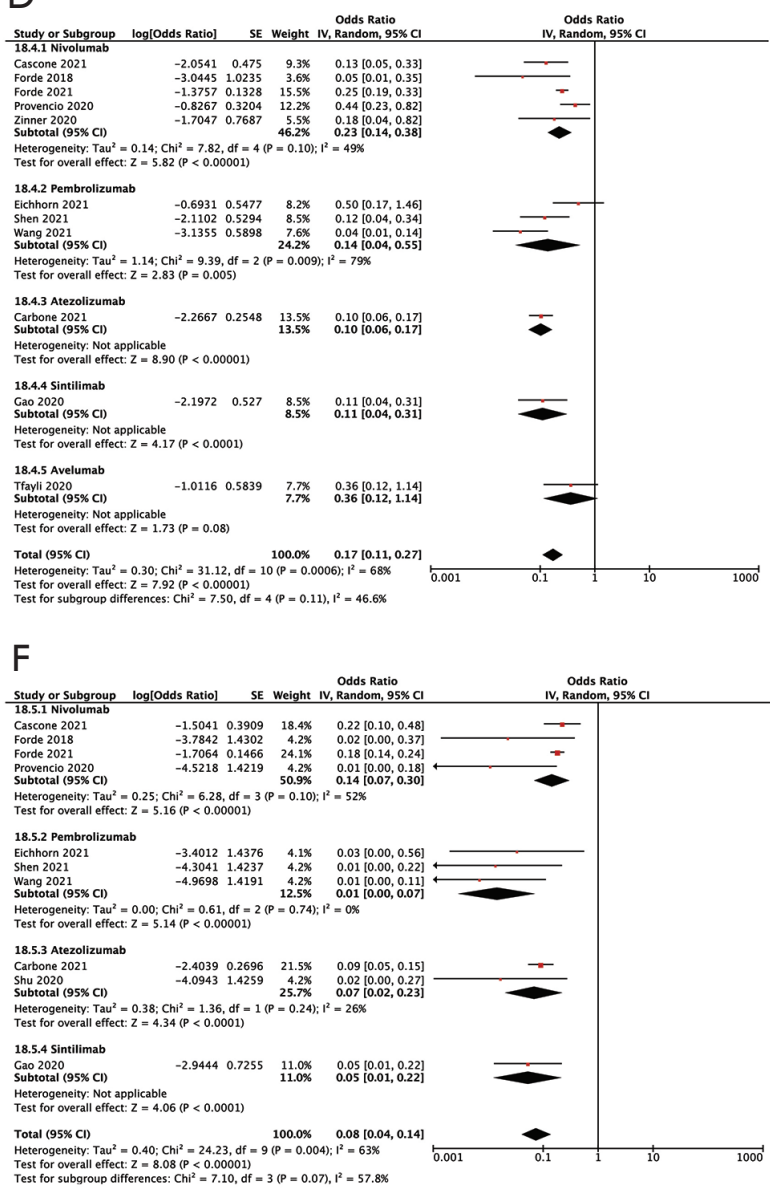

Figure 4 Forest plot of the efficacy and safety of subgroup analysis based on different ICI types. (A) MPR rate; (B) pCR rate; (C) incidence of TRAEs; (D) incidence of SAEs; (E) resection rate; (F) surgical delay rate. MPR, major pathological response; pCR, complete pathological response; TRAE, treatment-related adverse event; SAE, severe adverse event; SE, standard error; IV, inverse variance; CI, confidence interval; ICIs, immune checkpoint inhibitors. 

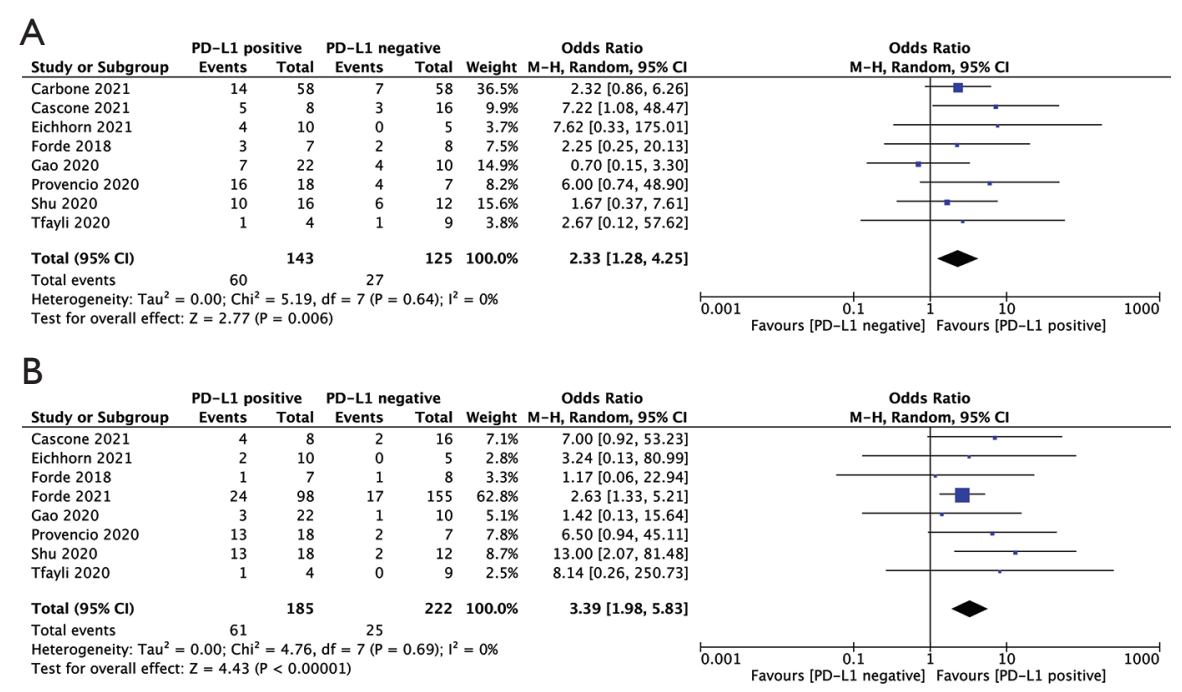

Figure 5 Forest plot of the pathological response of subgroup analysis based on PD-L1 expression. (A) MPR among patients with negative or positive PD-L1 expression; (B) pCR among patients with negative or positive PD-L1 expression. PD-L1 negative, PD-L1 TPS <1\%; PD-L1 positive, PD-L1 TPS $\geq 1 \%$; MPR, major pathological response; pCR, complete pathological response; M-H, Mantel-Haenszel; CI, confidence interval; PD-L1, programmed death-ligand 1.

those who received nivolumab-based neoadjuvant therapy were more likely [12.3\% (95\% CI: $6.5-23.1 \%$ )] to have surgical delay. Overall, there were no significant findings on the correlations between ICI types and the efficacy or safety of neoadjuvant immunotherapy.

\section{PD-L1 expression, histology subtypes and smoking status}

Next, we explored the potential effect of PD-L1 expression, histology subtypes and smoking status on efficacy of neoadjuvant immunotherapy or chemoimmunotherapy. As shown in Figure 5, patients with positive PD-L1 expression [defined as tumor proportion score (TPS) $\geq 1 \%$ ] showed significantly improved MPR (OR 2.33, 95\% CI: 1.28-4.25, $\mathrm{P}=0.006$ ) and pCR rates (OR 3.39, 95\% CI: 1.98-5.83, $\mathrm{P}<0.001$ ) than those PD-L1 negative patients (defined as TPS $<1 \%$ ). Neither squamous cell carcinoma nor smoking status had significant effects on MPR and pCR rates (Figure S3). But smokers showed a tendency of better MPR rate than nonsmokers, without statistical significance found (OR 2.18, 95\% CI: 0.84-5.65, P=0.11). Analyses of resection rate, incidence of TRAEs and SAEs were not performed in these subgroups, due to no available data provided in enrolled studies.

\section{Sensitivity analysis}

Our subgroup analysis data suggested that different neoadjuvant treatment modes were one of the main sources of heterogeneity. Subsequently, to ensure that the combined results were not severely swayed by certain trials, we performed sensitivity analysis for studies within the monoICI subgroup and ICI plus chemotherapy subgroup. As shown in Figure S4, the Carbone 2021 study (14) was the main source of statistical heterogeneity in the meta-analysis for MPR rate in the mono-ICI subgroup. After removing this outlier study, there was no heterogeneity among the remaining studies $\left(\mathrm{P}=0.70, \mathrm{I}^{2}=0 \%\right)$.

For studies included in the ICI plus chemotherapy subgroup (namely the chemoimmunotherapy subgroup), significant heterogeneity was observed in the meta-analysis for all endpoints (Figure 3). Therefore, a sensitivity analysis was performed, in which 1 study was removed at a time, to evaluate whether the results could have been significantly affected by a single study (Figure S5). We found that the removal of 3 studies decreased the heterogeneity for the metaanalysis of MPR and pCR rates (Figure S5A,S5B). Removal of any of these studies at a time did not significantly change our results (Table S1). For the incidence of TRAEs, only 3 studies were included in the ICI plus chemotherapy subgroup. Removing the Forde 2021 (25) or Provencio 2020 (28) study markedly reduced the heterogeneity, but the latter one affected the pooled incidence of TRAEs (Figure S5C). The Wang 2021 study (21) made the largest contribution to heterogeneity for the incidence of SAEs (Figure S5D). The 
Yang 2018 study (24) and Forde 2021 study (25) were the most significant contributors to heterogeneity in the metaanalysis of resection rate and surgical delay rate, respectively (Figure S5E,S5F). As shown in Table S1, after removal of any of the above studies, the MPR and pCR rates of the ICI plus chemotherapy subgroup were consistently higher than those of the mono-ICI subgroup, and the incidence of SAEs, resection rate, and surgical delay rate appeared to be comparable to the mono-ICI subgroup, confirming the stability of our results.

\section{Profiles of TRAEs and SAEs in patients who received neoadjuvant immunotherapy or chemoimmunotherapy}

We further summarized the profiles of TRAEs and SAEs after neoadjuvant immunotherapy or chemoimmunotherapy according to different organ systems. There were 11 studies $(\mathrm{n}=367)$ reporting the profiles of TRAEs and 12 studies $(\mathrm{n}=380)$ reporting the profiles of SAEs. As shown in Figure 6A, TRAEs (any grade) with incidence $>10 \%$ included fatigue $(32.2 \%)$, alopecia $(27.2 \%)$, anorexia (22.3\%), nausea $(18.0 \%)$, rash (16.9\%), anemia (14.4\%), neutropenia (13.4\%), and diarrhea (10.4\%). The most common SAEs which occurred in NSCLC patients who received neoadjuvant immunotherapy or chemoimmunotherapy included neutropenia (5.5\%), increased liver enzymes (1.8\%), pneumonitis (1.3\%), rash $(1.1 \%)$, thrombocytopenia $(1.1 \%)$, and febrile neutropenia (1.1\%) (Figure 6B).

\section{Discussion}

In this study, we conducted a meta-analysis of 16 studies focusing on neoadjuvant immunotherapy or chemoimmunotherapy in resectable NSCLC. Our results supported the efficacy and safety of neoadjuvant immunotherapy and chemoimmunotherapy. More importantly, we found that neoadjuvant chemoimmunotherapy had significantly higher MPR and pCR rates than neoadjuvant single-agent immunotherapy, without increasing the incidence of SAEs or surgical delay in patients with NSCLC.

Over the past decades, numerous studies have been conducted to optimize the treatment modalities for NSCLC, but the 5 -year survival rate of patients remains poor. Despite surgery and adjuvant therapy, about $20-30 \%$ of patients with stage I, $50 \%$ of patients with stage II, and $60 \%$ of patients with stage IIIA NSCLC die within 5 years (34). In recent years, an increased number of clinical trials have reported the efficacy and safety of neoadjuvant immunotherapy or chemoimmunotherapy in NSCLC using different treatment modes and various ICIs. Results from a metaanalysis by Jia et al. (33) published in 2020 supported the efficacy and safety of neoadjuvant immunotherapy in stages I-III NSCLC. However, only 7 studies and 252 patients were included, among which only 1 study (CheckMate 159 trial) provided complete published data, while the other 6 studies were all ongoing. Limited research was included in this meta-analysis, making it difficult to reveal differences among different treatment modes and ICI types. So far, more data have been released from a series of clinical trials. Thus, it is necessary to perform a meta-analysis with newly updated research data.

Our results showed that the pooled MPR rate and pCR rate of neoadjuvant immunotherapy and chemoimmunotherapy were $43.5 \%$ and $21.9 \%$, respectively. These rates were remarkably higher if using previous published data on neoadjuvant chemotherapy as a historical control which showed a pooled pCR rate of 4\% (9). Updated data from the CheckMate 816 trial (25) also demonstrated the advantages of neoadjuvant chemoimmunotherapy over chemotherapy alone in NSCLC by showing a significantly higher MPR rate $(37 \%$ vs. $9 \%)$ and $\mathrm{pCR}$ rate $(24 \%$ vs. $2 \%)$ in the chemoimmunotherapy arm. Although the median overall survival and recurrence-free survival were not reached in the studies of our meta-analysis, clinical benefits in survival outcomes can be expected based on the association of MPR or pCR with improved survival $(10,35)$. On the other hand, it has been reported that chemotherapy can enhance the efficacy of immunotherapy by increasing tumor antigen presentation $(36,37)$. However, whether the combination of immunotherapy and chemotherapy before surgery has a synergistic effect remains unclear. Interestingly, in the subgroup analysis based on neoadjuvant treatment modes, the MPR rate and pCR rate of the ICI plus chemotherapy subgroup were higher than those of the mono-ICI subgroup. Meanwhile, dual ICIs of nivolumab plus ipilimumab exhibited a high pCR rate comparable to neoadjuvant chemoimmunotherapy. These data consistently demonstrate that neoadjuvant chemoimmunotherapy and dual ICIs are advantageous for the pathological response of NSCLC patients over single-agent immunotherapy or chemotherapy alone.

Different kinds of ICIs were used in the studies included in this meta-analysis. While these ICIs act against tumor cells through similar molecular mechanisms, they may result in different efficacy and safety profiles in NSCLC $(38,39)$. Our subgroup analysis based on ICI types suggested 

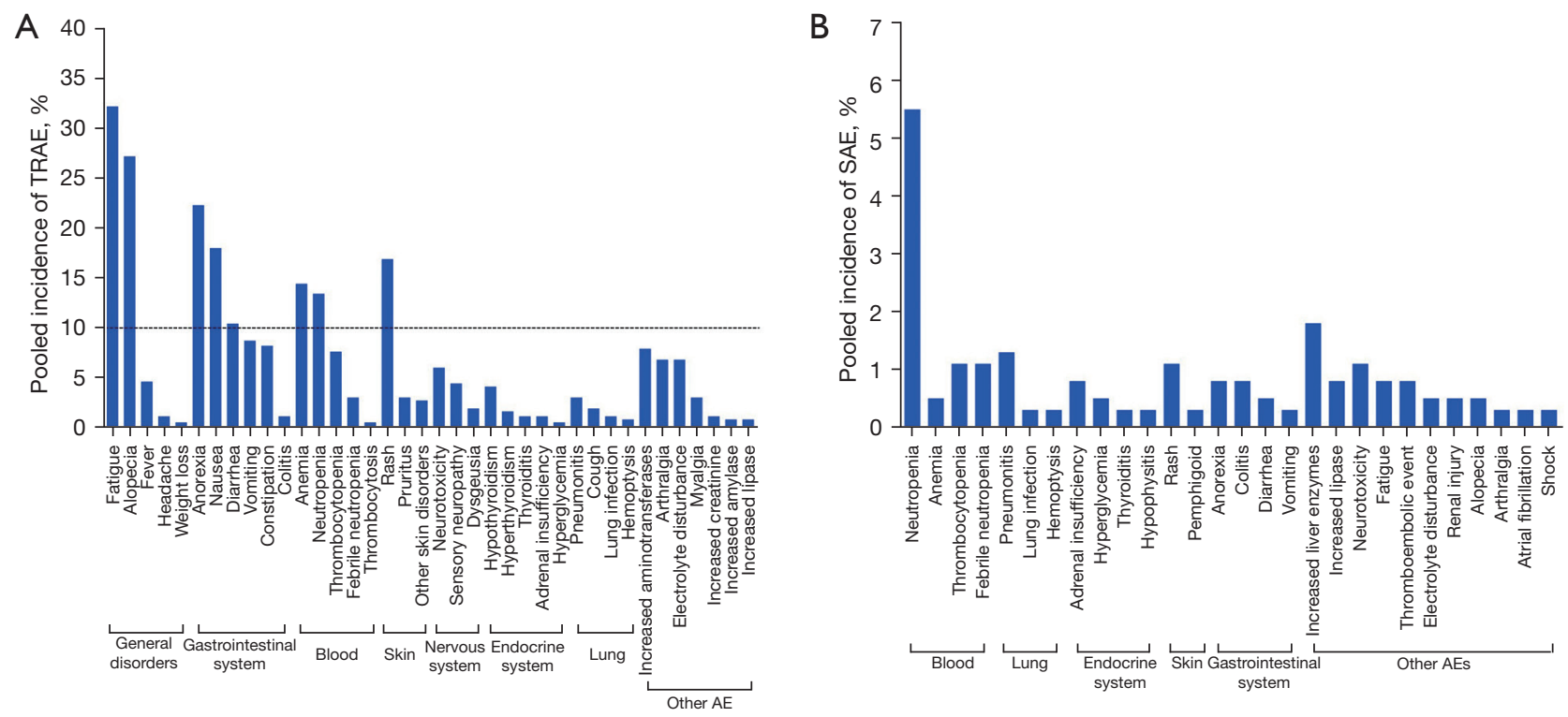

Figure 6 Pooled incidence of TRAEs and SAEs reported in clinical trials. TRAE, treatment-related adverse event; SAE, severe adverse event.

that there is no evidence for a dominant or superior ICI in neoadjuvant immunotherapy or chemoimmunotherapy at present. However, nivolumab- and pembrolizumab-based neoadjuvant therapy showed a higher MPR rate (nivolumab $51.5 \%$, pembrolizumab $46.8 \%$ ) and pCR rate (nivolumab $29.1 \%$, pembrolizumab $31.5 \%$ ), while avelumab-based neoadjuvant therapy had a relatively lower MPR rate $(20.0 \%)$ and pCR rate $(6.5 \%)$. Furthermore, the pooled MPR rate was similar among anti-PD-1 agents (nivolumab $51.5 \%$, pembrolizumab $46.8 \%$ ) and anti-PD-L1 agents (durvalumab $48.5 \%$, atezolizumab $33.3 \%$ ), but the pooled pCR rate for neoadjuvant regimens using anti-PD-1 agents (nivolumab 29.1\%, pembrolizumab 31.5\%) was significantly higher than those using anti-PD-L1 agents (durvalumab $14.5 \%$, atezolizumab $14.5 \%$ ).

Clinical and histopathological characteristics of NSCLC patients affect the efficacy of immunotherapy. PD-L1 TPS is a well-recognized biomarker for response to ICIs based on abundant clinical research data (40). Single-agent pembrolizumab has been recommended as the first-line treatment in stage IV NSCLC patients with PD-L1 TPS $\geq 50 \%$ and without EGFR/ALK aberrations. However, potential effect of PD-L1 TPS on efficacy of neoadjuvant immunotherapy remains unclear. In this meta-analysis, our data demonstrate that patients with PD-L1 TPS $\geq 1 \%$ have a favorable pathological response to neoadjuvant ICIs either combined with chemotherapy or not. It suggests that
PD-L1 TPS may be a useful biomarker of MPR or overall response rate in the era of neoadjuvant immunotherapy, which needs more clinical studies to confirm.

It has been observed that patients with stage III NSCLC appear to have a better response to neoadjuvant chemotherapy than those with stage I or II disease (41). Whether a similar phenomenon exists in neoadjuvant immunotherapy or chemoimmunotherapy remains unknown. In the NADIM trial and SAKK 16/14 trial, which exclusively enrolled patients with stage III NSCLC, the MPR rate after neoadjuvant chemoimmunotherapy appeared higher than other trials. The NADIM trial reported a remarkable pCR rate of $57 \%$. However, the pCR rates reported in the SAKK $16 / 14$ trial and another prospective single-arm study in stage IIIA NSCLC (21) were $15 \%$ and $29 \%$, respectively, which did not support this point.

The key findings of this meta-analysis also include the safety of neoadjuvant immunotherapy or chemoimmunotherapy prior to surgery without significant surgical delays. In a previous meta-analysis including 36 phase II/III clinical trials, the pooled incidence for all-grade irAEs was estimated to range between $54 \%$ and $76 \%$ in cancers, included lung cancer, melanoma, urinary system cancer, head and neck cancer, and digestive system cancer (39). For patients with lung cancer, Berti et al. (42) reported that the incidence of all-grade irAEs and severe irAEs for ICIs were $37.1 \%$ and $18.5 \%$, respectively. It is recognized that 
the incidence of TRAEs or SAEs significantly increases if ICIs are combined with chemotherapy (43). Therefore, the safety of neoadjuvant chemoimmunotherapy has attracted significant attention. In the present meta-analysis, only 5 trials enrolled NSCLC patients who received mono-ICIs before surgery, while the others were either combined with chemotherapy or ipilimumab. Thus, the incidence of TRAEs after neoadjuvant therapy was used as a major safety-related endpoint, rather than irAEs. Our results showed that the pooled incidence of TRAEs in the 16 studies was $54.8 \%$, which seemed higher than previously published data of neoadjuvant immunotherapy. The inclusion of patients who received chemoimmunotherapy could explain this difference. Interestingly, while subgroup analysis demonstrated that the combination of chemotherapy before surgery increased the incidence of TRAEs $(73.9 \%$ vs. $42.9 \%)$, it did not significantly increase the incidence of SAEs (18.0\% vs. $12.3 \%, \mathrm{P}=0.32)$. Besides, the incidence of SAEs seemed similar between mono-ICI and dual ICIs subgroups (12.3\% vs. 9.9\%). Compared with patients who received immunotherapy, the pooled resection rate was similar and the surgical delay rate was even lower in those who received neoadjuvant chemoimmunotherapy. Therefore, our data demonstrates that both neoadjuvant immunotherapy and chemoimmunotherapy are tolerable for patients with NSCLC.

Fibrosis and adhesion in the chest cavity have been reported after neoadjuvant ICIs, which could potentially increase the risk of conversion from minimally invasive surgery to thoracotomy during the operation. In this metaanalysis, only 3 studies provided data on conversion, and the pooled conversion rate was $17.4 \%$. Notably, the conversion rate in the Forde 2018 study (13) was higher than the other 2 studies [Duan 2021 (22), Yang 2018 (24)], which suggests that chemoimmunotherapy before surgery may help reduce surgical complexity compared with neoadjuvant immunotherapy alone. However, more randomized clinical trials are required to further confirm these observations.

Heterogeneity cannot be ignored in this meta-analysis. Our subgroup analysis demonstrated that different treatment modes are a main source of heterogeneity. After subgroup analysis based on treatment modes, heterogeneity in the mono-ICI subgroup markedly reduced. However, significant heterogeneity was still observed within the ICI plus chemotherapy subgroup. Multiple factors may contribute to the heterogeneity, including clinical staging, histology subtypes, PD-L1 expression level, smoking status and ethnicity of patients. These clinical trials were conducted in America, Asia and Europe, and enrolled patients from different ethnic groups. While most trials enrolled patients with stages I-III NSCLC, some were focused on stage III disease. The proportions of squamous cell carcinoma in these studies were also markedly different, ranging from $13 \%$ to $100 \%$. PD-L1 TPS of patients and proportion of smokers also varied in these studies. Diversity in patient characteristics may explain the large heterogeneity in the incidence of TRAEs in the chemoimmunotherapy subgroup. ICI types can also be an important source of heterogeneity. Varying degrees of differences in endpoints were shown in subgroups of different ICIs, even though no statistical significance was seen in the subgroup analysis, which may be explained by the small sample size. Furthermore, study design and quality may also affect the homogeneity of these studies. Only 2 were randomized clinical trials, while the others were phase 1 or 2 single-arm studies. Removal of 1 study at a time could not eliminate the heterogeneity in this meta-analysis. However, based on the data of the sensitivity analysis, removal of any study did not significantly change our results or affect the overall conclusion. The results of sensitivity analyses for different endpoints were consistent (except for TRAEs for the chemoimmunotherapy subgroup), leading to solid conclusions.

There are several limitations in this meta-analysis. Firstly, although 16 studies were included, most were nonrandomized single-arm clinical trials with a small sample size. As a consequence, this set of comparative analyses were based on indirect comparisons. Secondly, not all the included studies provided data on all key endpoints, and some have not yet reached their endpoint. Therefore, complete patient data for these studies are not available at present. Furthermore, the follow-up periods of these studies were relatively short, with limited data reporting on survival outcomes. With the rapid growth in the number of active trials on neoadjuvant immunotherapy or chemoimmunotherapy, we believe that more conclusive meta-analyses can be conducted by including more largescale multicenter randomized clinical trials.

In conclusion, this meta-analysis demonstrated the efficacy and safety of neoadjuvant immunotherapy and chemoimmunotherapy. Neoadjuvant chemoimmunotherapy provide a significant improvement in pathological response versus single-agent immunotherapy, without increasing the incidence of SAEs or surgical delay. Neoadjuvant dual ICIs of nivolumab and ipilimumab also achieve an impressive pCR rate with tolerable toxicity, which needs further 
confirmation by more clinical trials. Our data support the potential application of neoadjuvant immunotherapy or chemoimmunotherapy for resectable stages I-III disease. However, the effect on long term survival and recurrence is yet to be determined. In addition, there are more frequent TRAEs and SAEs related to chemoimmunotherapy even though significance was not found in our meta-analysis. Therefore, neoadjuvant therapy regimens should be chosen with caution based on comprehensive evaluation of individual benefits and risks when implementing in clinical practice.

\section{Acknowledgments}

The authors appreciate the academic support from the AME Lung Cancer Collaborative Group.

Funding: This study was supported by Major Science and Technology Project of Hunan Province (2019SK2251 to ML), Natural Science Foundation of China (81903020 to ML), China Postdoctoral Science Foundation (2019M652812 to ML), and National Multidisciplinary Cooperative Diagnosis and Treatment Capacity Building Project for Major Diseases (Lung Cancer, z027002).

\section{Footnote}

Reporting Checklist: The authors have completed the PRISMA reporting checklist. Available at https://tlcr. amegroups.com/article/view/10.21037/tlcr-22-75/rc

Conflicts of Interest: All authors have completed the ICMJE uniform disclosure form (available at https://tlcr.amegroups. com/article/view/10.21037/tlcr-22-75/coif). Prof. SY serves as an unpaid editorial board member of Translational Lung Cancer Research from October 2021 to September 2023. The other authors have no conflicts of interest to declare.

Ethical Statement: The authors are accountable for all aspects of the work in ensuring that questions related to the accuracy or integrity of any part of the work are appropriately investigated and resolved.

Open Access Statement: This is an Open Access article distributed in accordance with the Creative Commons Attribution-NonCommercial-NoDerivs 4.0 International License (CC BY-NC-ND 4.0), which permits the noncommercial replication and distribution of the article with the strict proviso that no changes or edits are made and the original work is properly cited (including links to both the formal publication through the relevant DOI and the license). See: https://creativecommons.org/licenses/by-nc-nd/4.0/.

\section{References}

1. Siegel RL, Miller KD, Jemal A. Cancer statistics, 2020. CA Cancer J Clin 2020;70:7-30.

2. Zappa C, Mousa SA. Non-small cell lung cancer: current treatment and future advances. Transl Lung Cancer Res 2016;5:288-300.

3. Duma N, Santana-Davila R, Molina JR. Non-Small Cell Lung Cancer: Epidemiology, Screening, Diagnosis, and Treatment. Mayo Clin Proc 2019;94:1623-40.

4. Uprety D, Mandrekar SJ, Wigle D, et al. Neoadjuvant Immunotherapy for NSCLC: Current Concepts and Future Approaches. J Thorac Oncol 2020;15:1281-97.

5. Blumenthal GM, Bunn PA Jr, Chaft JE, et al. Current Status and Future Perspectives on Neoadjuvant Therapy in Lung Cancer. J Thorac Oncol 2018;13:1818-31.

6. Pisters KM, Vallières E, Crowley JJ, et al. Surgery with or without preoperative paclitaxel and carboplatin in earlystage non-small-cell lung cancer: Southwest Oncology Group Trial S9900, an intergroup, randomized, phase III trial. J Clin Oncol 2010;28:1843-9.

7. Scagliotti GV, Pastorino U, Vansteenkiste JF, et al. Randomized phase III study of surgery alone or surgery plus preoperative cisplatin and gemcitabine in stages IB to IIIA non-small-cell lung cancer. J Clin Oncol 2012;30:172-8.

8. Ren $\mathrm{S}, \mathrm{Xu} \mathrm{A}, \mathrm{Lin} \mathrm{Y}$, et al. A narrative review of primary research endpoints of neoadjuvant therapy for lung cancer: past, present and future. Transl Lung Cancer Res 2021;10:3264-75.

9. NSCLC Meta-analysis Collaborative Group. Preoperative chemotherapy for non-small-cell lung cancer: a systematic review and meta-analysis of individual participant data. Lancet 2014;383:1561-71.

10. Hellmann MD, Chaft JE, William WN Jr, et al. Pathological response after neoadjuvant chemotherapy in resectable non-small-cell lung cancers: proposal for the use of major pathological response as a surrogate endpoint. Lancet Oncol 2014;15:e42-50.

11. Kang J, Zhang C, Zhong WZ. Neoadjuvant immunotherapy for non-small cell lung cancer: State of the art. Cancer Commun (Lond) 2021;41:287-302.

12. Mencoboni M, Ceppi M, Bruzzone M, et al. Effectiveness and Safety of Immune Checkpoint Inhibitors for Patients 
with Advanced Non Small-Cell Lung Cancer in RealWorld: Review and Meta-Analysis. Cancers (Basel) 2021;13:1388.

13. Forde PM, Chaft JE, Smith KN, et al. Neoadjuvant PD-1 Blockade in Resectable Lung Cancer. N Engl J Med 2018;378:1976-86.

14. Carbone D, Lee J, Kris M, et al. Clinical/Biomarker Data for Neoadjuvant Atezolizumab in Resectable Stage IB-IIIB NSCLC: Primary Analysis in the LCMC3 Study. Journal of Thoracic Oncology 2021;16:S115-S116.

15. Gao S, Li N, Gao S, et al. Neoadjuvant PD-1 inhibitor (Sintilimab) in NSCLC. J Thorac Oncol 2020;15:816-26.

16. Eichhorn F, Klotz LV, Kriegsmann M, et al. Neoadjuvant anti-programmed death-1 immunotherapy by pembrolizumab in resectable non-small cell lung cancer: First clinical experience. Lung Cancer 2021;153:150-7.

17. Bott MJ, Yang SC, Park BJ, et al. Initial results of pulmonary resection after neoadjuvant nivolumab in patients with resectable non-small cell lung cancer. J Thorac Cardiovasc Surg 2019;158:269-76.

18. Cascone T, William WN Jr, Weissferdt A, et al. Neoadjuvant nivolumab or nivolumab plus ipilimumab in operable non-small cell lung cancer: the phase 2 randomized NEOSTAR trial. Nat Med 2021;27:504-14.

19. Shu CA, Gainor JF, Awad MM, et al. Neoadjuvant atezolizumab and chemotherapy in patients with resectable non-small-cell lung cancer: an open-label, multicentre, single-arm, phase 2 trial. Lancet Oncol 2020;21:786-95.

20. Shen D, Wang J, Wu J, et al. Neoadjuvant pembrolizumab with chemotherapy for the treatment of stage IIB-IIIB resectable lung squamous cell carcinoma. J Thorac Dis 2021;13:1760-8.

21. Wang J, Li J, Cai L, et al. The safety and efficacy of neoadjuvant programmed death 1 inhibitor therapy with surgical resection in stage IIIA non-small cell lung cancer. Ann Transl Med 2021;9:486.

22. Duan H, Wang T, Luo Z, et al. Neoadjuvant programmed cell death protein 1 inhibitors combined with chemotherapy in resectable non-small cell lung cancer: an open-label, multicenter, single-arm study. Transl Lung Cancer Res 2021;10:1020-8.

23. Tfayli A, Al Assaad M, Fakhri G, et al. Neoadjuvant chemotherapy and Avelumab in early stage resectable nonsmall cell lung cancer. Cancer Med 2020;9:8406-11.

24. Yang CJ, McSherry F, Mayne NR, et al. Surgical Outcomes After Neoadjuvant Chemotherapy and Ipilimumab for Non-Small Cell Lung Cancer. Ann Thorac Surg 2018;105:924-9.
25. Forde PM, Spicer J, Lu S, et al. Nivolumab (NIVO) + platinum-doublet chemotherapy (chemo) vs chemo as neoadjuvant treatment (tx) for resectable (IB-IIIA) nonsmall cell lung cancer (NSCLC) in the phase 3 CheckMate 816 trial. In: Proceedings of the 112th Annual Meeting of the American Association for Cancer Research; 2021 April 10-15. Philadelphia (PA): AACR; 2021.

26. Rothschild SI, Zippelius A, Eboulet EI, et al. SAKK 16/14: Durvalumab in Addition to Neoadjuvant Chemotherapy in Patients With Stage IIIA(N2) Non-Small-Cell Lung Cancer-A Multicenter Single-Arm Phase II Trial. J Clin Oncol 2021;39:2872-80.

27. Zinner R, Axelrod R, Solomides CC, et al. Neoadjuvant nivolumab $(\mathrm{N})$ plus cisplatin $(\mathrm{C}) /$ pemetrexed $(\mathrm{P})$ or cisplatin /gemcitabine (G) in resectable NSCLC. J Clin Oncol 2020;38:9051.

28. Provencio M, Nadal E, Insa A, et al. Neoadjuvant chemotherapy and nivolumab in resectable non-small-cell lung cancer (NADIM): an open-label, multicentre, singlearm, phase 2 trial. Lancet Oncol 2020;21:1413-22.

29. Waldman AD, Fritz JM, Lenardo MJ. A guide to cancer immunotherapy: from $T$ cell basic science to clinical practice. Nat Rev Immunol 2020;20:651-68.

30. Michot JM, Bigenwald C, Champiat S, et al. Immunerelated adverse events with immune checkpoint blockade: a comprehensive review. Eur J Cancer 2016;54:139-48.

31. Ramos-Casals M, Brahmer JR, Callahan MK, et al. Immune-related adverse events of checkpoint inhibitors. Nat Rev Dis Primers 2020;6:38.

32. Moher D, Liberati A, Tetzlaff J, et al. Preferred reporting items for systematic reviews and meta-analyses: the PRISMA statement. BMJ 2009;339:b2535.

33. Jia XH, Xu H, Geng LY, et al. Efficacy and safety of neoadjuvant immunotherapy in resectable nonsmall cell lung cancer: A meta-analysis. Lung Cancer 2020;147:143-53.

34. Goldstraw P, Chansky K, Crowley J, et al. The IASLC Lung Cancer Staging Project: Proposals for Revision of the TNM Stage Groupings in the Forthcoming (Eighth) Edition of the TNM Classification for Lung Cancer. J Thorac Oncol 2016;11:39-51.

35. Pataer A, Kalhor N, Correa AM, et al. Histopathologic response criteria predict survival of patients with resected lung cancer after neoadjuvant chemotherapy. J Thorac Oncol 2012;7:825-32.

36. Ramakrishnan R, Gabrilovich DI. Novel mechanism of synergistic effects of conventional chemotherapy and immune therapy of cancer. Cancer Immunol Immunother 
2013;62:405-10.

37. Emens LA, Middleton G. The interplay of immunotherapy and chemotherapy: harnessing potential synergies. Cancer Immunol Res 2015;3:436-43.

38. Pillai RN, Behera M, Owonikoko TK, et al. Comparison of the toxicity profile of PD-1 versus PD-L1 inhibitors in non-small cell lung cancer: A systematic analysis of the literature. Cancer 2018;124:271-7.

39. Xu C, Chen YP, Du XJ, et al. Comparative safety of immune checkpoint inhibitors in cancer: systematic review and network meta-analysis. BMJ 2018;363:k4226.

40. Camidge DR, Doebele RC, Kerr KM. Comparing and contrasting predictive biomarkers for immunotherapy and targeted therapy of NSCLC. Nat Rev Clin Oncol
2019;16:341-55.

41. Abratt RP, Lee JS, Han JY, et al. Phase II trial of gemcitabine-carboplatin-paclitaxel as neoadjuvant chemotherapy for operable non-small cell lung cancer. J Thorac Oncol 2006;1:135-40.

42. Berti A, Bortolotti R, Dipasquale M, et al. Meta-analysis of immune-related adverse events in phase 3 clinical trials assessing immune checkpoint inhibitors for lung cancer. Crit Rev Oncol Hematol 2021;162:103351.

43. Gandhi L, Rodríguez-Abreu D, Gadgeel S, et al. Pembrolizumab plus Chemotherapy in Metastatic NonSmall-Cell Lung Cancer. N Engl J Med 2018;378:2078-92.

(English Language Editor: C. Betlazar-Maseh)
Cite this article as: Jiang J, Wang Y, Gao Y, Sugimura H, Minervini F, Uchino J, Halmos B, Yendamuri S, Velotta JB, Li M. Neoadjuvant immunotherapy or chemoimmunotherapy in non-small cell lung cancer: a systematic review and meta-analysis. Transl Lung Cancer Res 2022;11(2):277-294. doi: 10.21037/ tlcr-22-75 


\section{Supplementary}

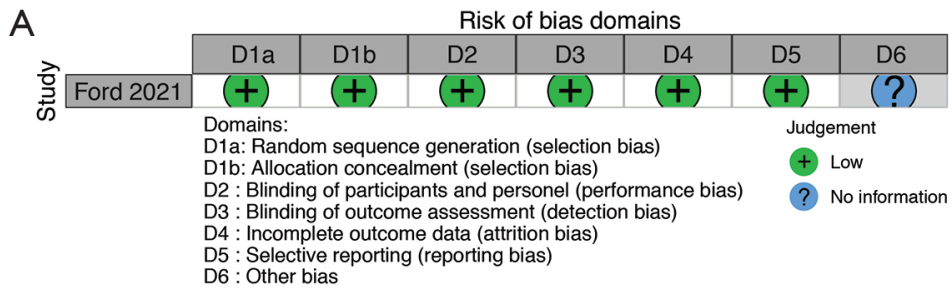

B

Risk of bias domains

\begin{tabular}{|c|c|c|c|c|c|c|c|c|}
\hline & D1 & D2 & D3 & D4 & D5 & D6 & D7 & Overall \\
\hline Carbone 2021 & $\oplus$ & $\oplus$ & $\oplus$ & $\oplus$ & $\oplus$ & $\oplus$ & $\oplus$ & ( \\
\hline Cascone 2021 & ( & + & + & + & + & + & + & ( \\
\hline Duan 2021 & ( & $\Theta$ & $\mathrm{x}$ & $\oplus$ & $\oplus$ & $\oplus$ & $\Theta$ & X \\
\hline Eichhorn 2021 & (t) & $\Theta$ & ( & ( & ( & ( & ( & $\Theta$ \\
\hline Forde 2018 & ( & $\Theta$ & ( & ( & ( & (+) & ( & - \\
\hline Gao 2020 & + & $\oplus$ & $\oplus$ & $\oplus$ & $\oplus$ & + & ( & $\oplus$ \\
\hline Provencio 2020 & ( & $\Theta$ & ( & $\oplus$ & $\oplus$ & $\oplus$ & $\oplus$ & $\Theta$ \\
\hline Rothchild 2020 & ? & $\Theta$ & $\oplus$ & $\oplus$ & ? & $\oplus$ & ? & ? \\
\hline Shen 2021 & 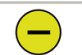 & $\Theta$ & ( & ( & $\Theta$ & ( & + & - \\
\hline Shu 2020 & \pm & $\oplus$ & ( & $\oplus$ & ( & $\oplus$ & ( & ( \\
\hline Tfayli 2020 & ( & - & 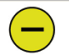 & $\oplus$ & $\oplus$ & $\oplus$ & $\oplus$ & - \\
\hline Wang 2021 & ( & - & $\oplus$ & $\oplus$ & (†) & $\oplus$ & - & $(-$ \\
\hline Yang 2018 & ( & ? & $\oplus$ & ( & $\Theta$ & - & $\Theta$ & ? \\
\hline Zinner 2020 & ? & ? & ( & $\oplus$ & ? & $\oplus$ & ? & ? \\
\hline & \multicolumn{6}{|c|}{ 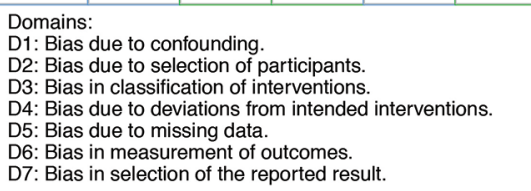 } & \multicolumn{2}{|c|}{$\begin{array}{l}\text { Judgement } \\
\text { \& Serious } \\
- \text { Moderate } \\
+ \text { Low } \\
? \text { ? No information }\end{array}$} \\
\hline
\end{tabular}

Figure S1 The risk of bias of the included studies. (A) Risk of bias of randomized trials; (B) Risk of bias of non-randomized trials. 
A

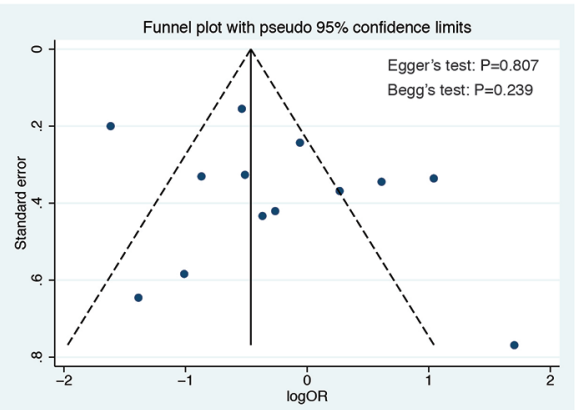

C

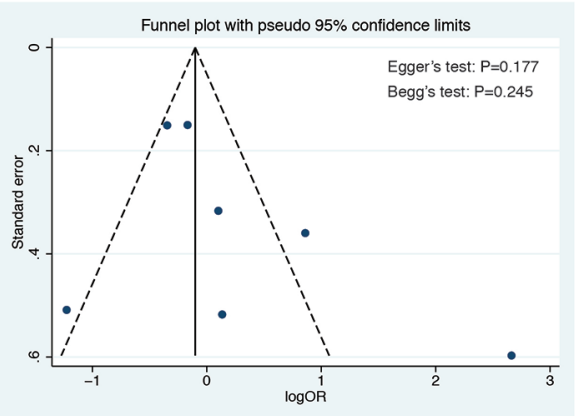

E

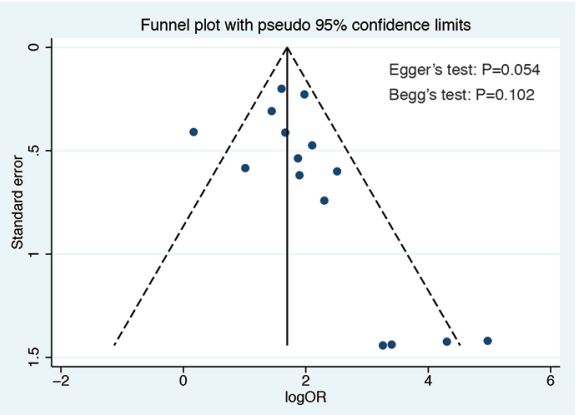

B

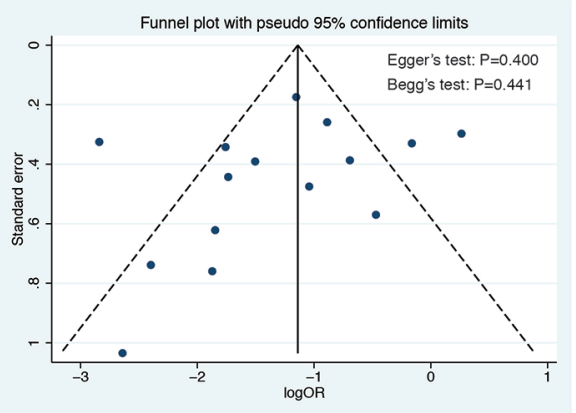

D

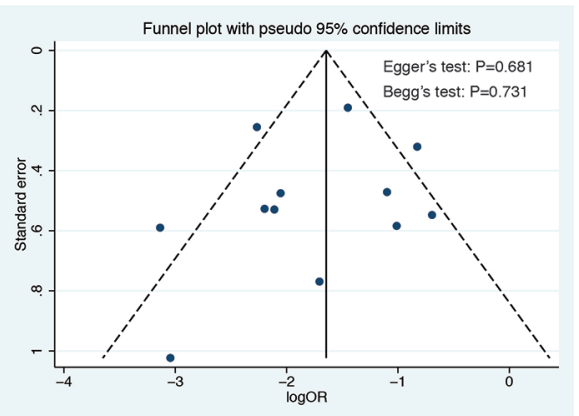

F

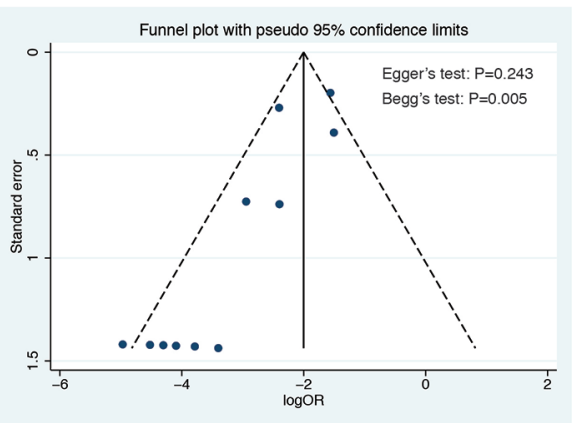

Figure S2 Publication bias of included studies. (A) Funnel plot for MPR; (B) Funnel plot for pCR; (C) Funnel plot for incidence of TRAE; (D) Funnel plot for incidence of SAE; (E) Funnel plot for resection rate; (F) Funnel plot for surgical delay rate. 


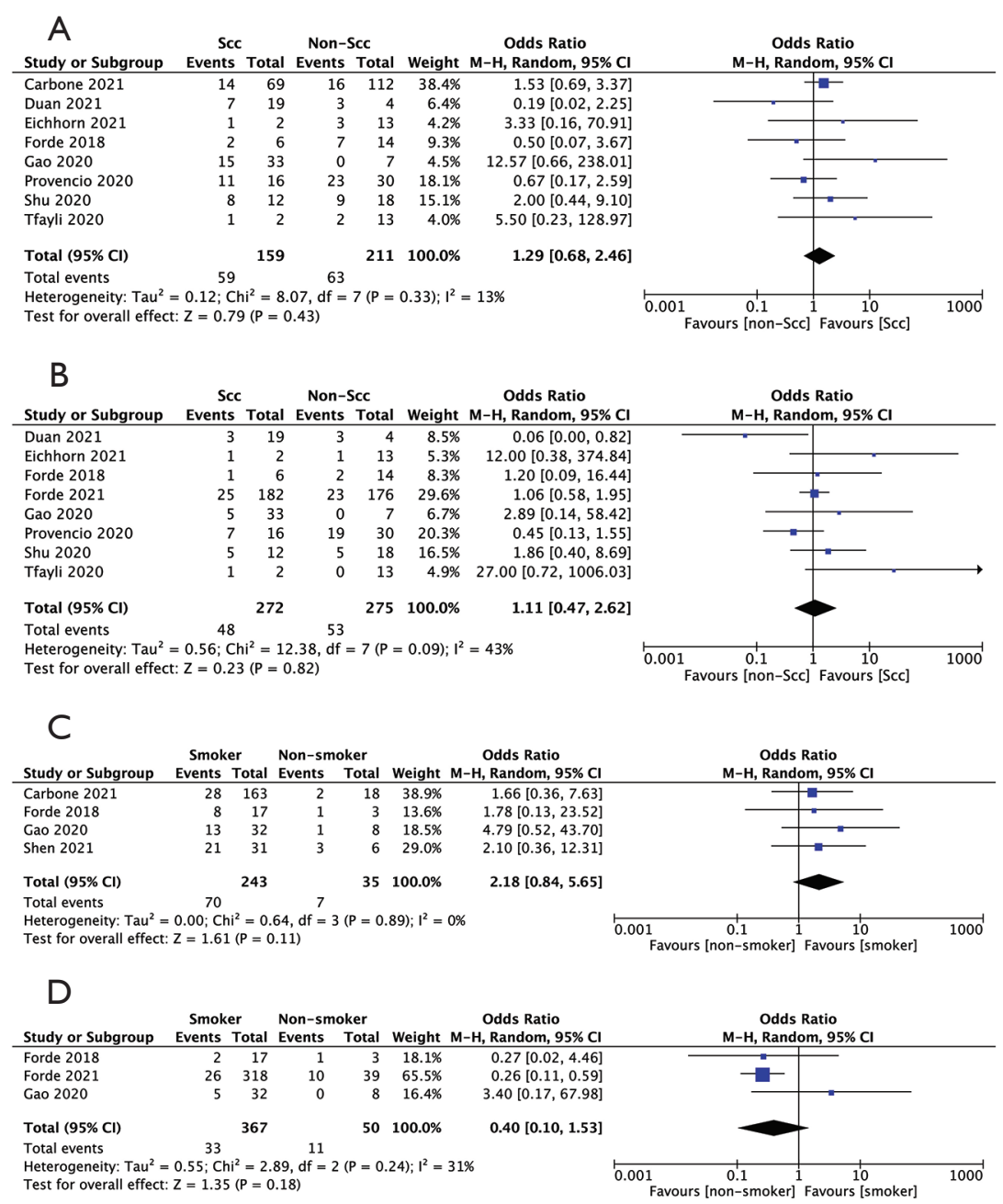

Figure S3 Forest plot of the pathological response of subgroup analysis based on histology subtypes and smoking status. (A) MPR among patients who are diagnosed with squamous cell carcinoma or non-squamous cell carcinoma; (B) pCR among patients diagnosed with squamous cell carcinoma or non-squamous cell carcinoma; (C) MPR among patients who are smokers or non-smokers; (D) pCR among patients who are smokers or non-smokers. Smokers, including current and former smokers; MPR, major pathological response; pCR, complete pathological response; $\mathrm{M}-\mathrm{H}$, Mantel-Haenszel; CI, confidence interval.

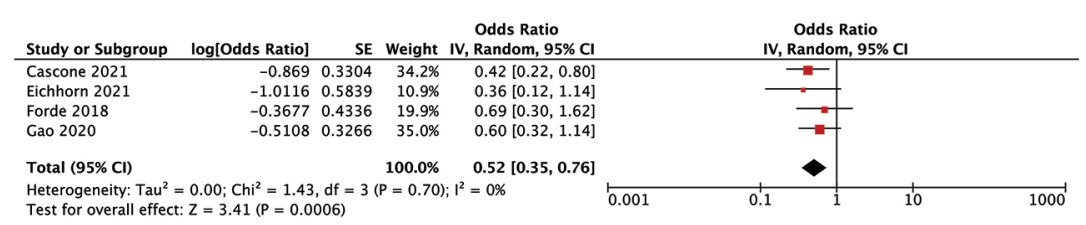

Figure S4 Forest plot of the MPR rate of the mono-ICI subgroup after removal of the Carbone 2021 study in the sensitivity analysis. MPR, major pathological response; ICI, immune checkpoint inhibitor. 
A
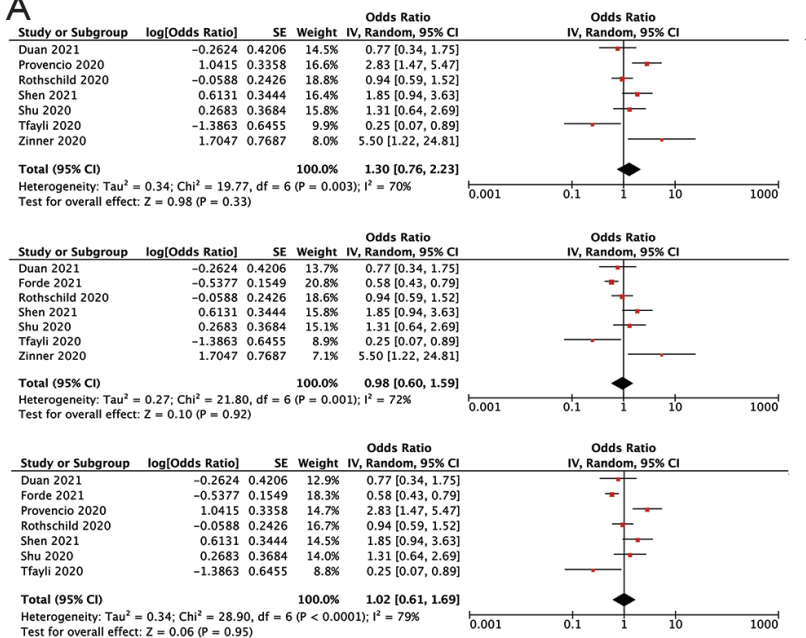

C

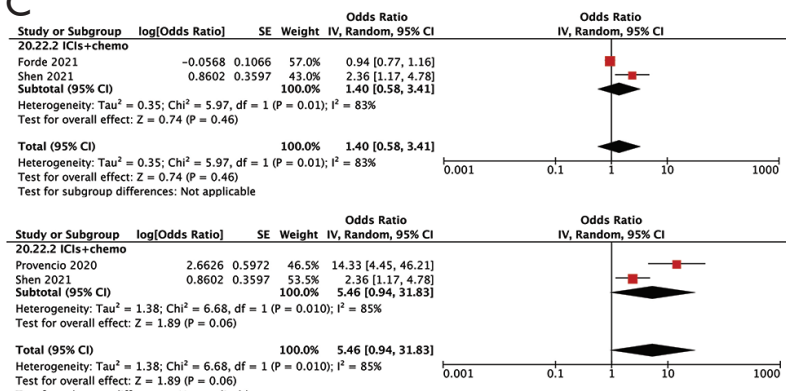

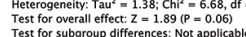

$\mathrm{E}$

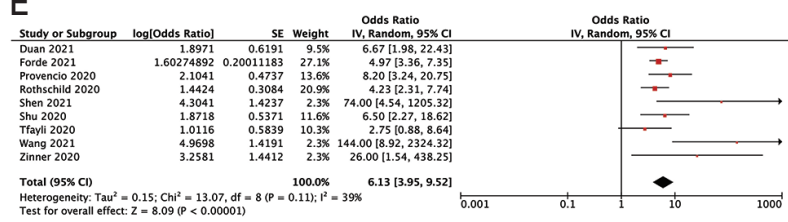

B
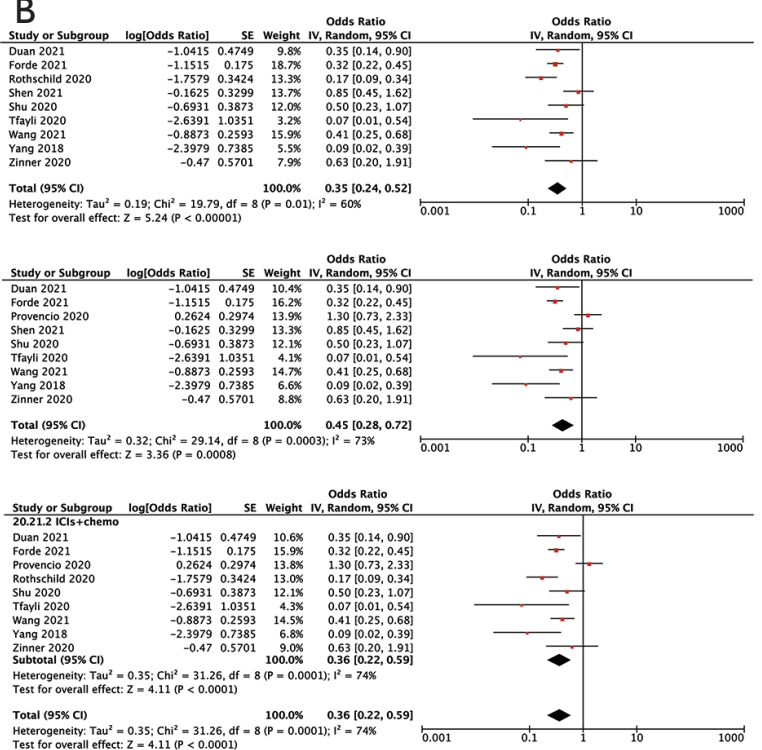

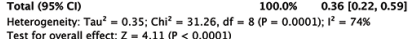

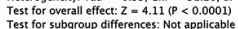

D

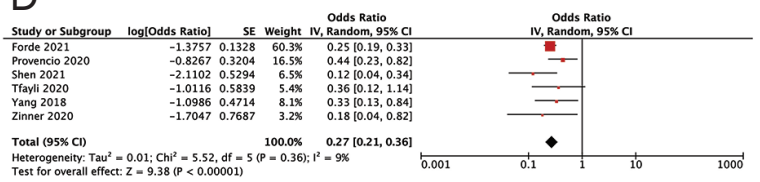

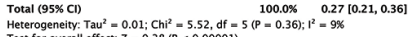

$\mathrm{F}$

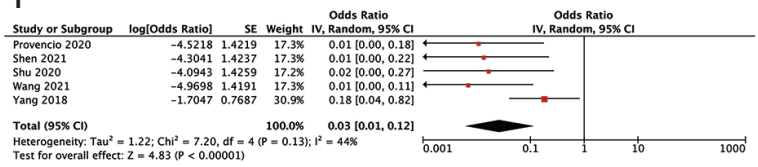

Figure S5 Forest plot of the efficacy and safety of the ICI + chemo subgroup in the sensitivity analysis. (A) MPR rate; (B) pCR rate; (C) Incidence of TRAEs; (D) incidence of SAEs; (E) resection rate; (F) surgical delay rate. MPR, major pathological response; pCR, complete pathological response; TRAE, treatment-related adverse event; SAE, severe adverse event; SE, standard error; IV, inverse variance; CI, confidence interval.

Table S1 Sensitivity analysis of efficacy- and safety-related endpoints

\begin{tabular}{|c|c|c|}
\hline Subgroup & Endpoints & Ratio $(95 \% \mathrm{Cl})$ \\
\hline Mono-ICl & MPR rate & $34.2(25.9-43.2)$ \\
\hline $\mathrm{ICl}+$ chemo & MPR rate & $56.5(43.2-69.0)^{\mathrm{a}} ; 49.5(37.5-61.4)^{\mathrm{b}} ; 50.5(37.9-62.8)^{\mathrm{c}}$ \\
\hline $\mathrm{ICI}+$ chemo & Incidence of TRAEs & $58.3(36.7-77.3)^{\mathrm{g}} ; 84.5(48.5-97.0)^{\mathrm{h}}$ \\
\hline $\mathrm{ICl}+$ chemo & Incidence of SAEs & $21.3(17.4-26.5)$ \\
\hline $\mathrm{ICl}+$ chemo & Surgical delay rate & $2.9(1.0-10.7)$ \\
\hline \multicolumn{3}{|c|}{$\begin{array}{l}\text { a, b }{ }^{c} \text { refer to the pooled MPR rate of the ICI + chemo subgroup after removal of the Forde } 2021 \text { study, Provencio } 2020 \text { study, or Zinner } \\
2020 \text { study, respectively; }{ }^{d},{ }^{e},{ }^{\dagger} \text { refer to the pooled pCR rate of the ICI + chemo subgroup after removal of the Provencio } 2020 \text { study, } \\
\text { Rothchild } 2021 \text { study, or Shen } 2021 \text { study, respectively; }{ }^{g},{ }^{h} \text { refer to the pooled incidence of TRAEs of the ICI + chemo subgroup after } \\
\text { removal of the Provencio } 2020 \text { study and Forde } 2021 \text { study, respectively. ICI, immune checkpoint inhibitor; chemo, chemotherapy; } \\
\text { mono-ICI, single-agent immune checkpoint inhibitor; MPR, major pathological response; pCR, complete pathological response; TRAE, } \\
\text { treatment-related adverse event; SAE, severe adverse event; Cl, confidence interval. }\end{array}$} \\
\hline
\end{tabular}

